\title{
Dates of entry and measures of imagery, concreteness, goodness, and familiarity for 1,046 words sampled from the Oxford English Dictionary
}

\author{
JOHN BENJAFIELD and RON MUCKENHEIM \\ Brock University, St. Catharines, Ontario, Canada
}

\begin{abstract}
The Oxford English Dictionary is the standard reference work for determining the earliest known instance of the occurrence of a word (its date of entry). Partly in order to facilitate research on the relation between the date of entry and other psychological variables, we gathered normative data on 1,046 words sampled from the Oxford English Dictionary. We present data for scales that measure the imagery, concreteness, goodness, and familiarity values for words. These norms may also be of use to researchers who are not explicitly concerned with words' date of entry, but who wish to sample words from a set that contains a large number of unfamiliar as well as familiar words.
\end{abstract}

Presented here is a new database consisting of norms for 1,046 words selected from the Oxford English Dictionary (OED). This database differs from existing databases in several ways. It is not restricted to nouns, as are the collections presented by Paivio, Yuille, and Madigan (1968) and Gilhooly and Logie (1980). Furthermore, it does not exclude uncommon, unfamiliar English words, as does the database in Friendly, Franklin, Hoffman, and Rubin (1982). Other unique features are pointed out below. In general, our database is designed to be representative of the words in the OED. Thus, the sample represents the types of words that occur in the English language, without regard to how frequently they occur.

This new database was created as part of an ongoing series of studies of the relationships between historical and psychological aspects of language (Benjafield, 1983, 1984, 1987a, 1987b; Benjafield \& Carson, 1985, 1986; Benjafield \& Muckenheim, in press a, in press b). Essentially we have been interested in the psychological correlates of the date at which a word is first recorded as having been used in the English language. The OED is the standard reference work for determining this latter variable, called date of entry. It consists of 12 volumes plus four supplements published between 1933 and 1986. This dictionary treats words historically, in each case giving a quotation that contains the earliest known instance of the occurrence of the word. The quotations are accompanied by dates, and it is these dates with which we have been primarily concerned.

Others have also investigated date of entry. Rubin (1980) obtained the dates of entry and several other

Preparation of this paper was supported by a grant from the Social Sciences and Humanities Research Council of Canada to the first author. We are grateful to Kris Frommhold and Dierk Muller for their assistance in organizing some of the data. Correspondence may be addressed to John Benjafield, Department of Psychology, Brock University, St. Catharines, Ontario L2S 3A1, Canada. measures for 125 words; in this paper our results will be compared to his. Williams (1983) has used dates of entry to explore the development of culturally important concepts. Olson and Astington (1986) have examined dates of entry in relation to problems of language development and literacy.

In our own work, we have been investigating some established psychological features of language-but from a relatively unexplored perspective, which was first suggested by Piaget (1950; see Flavell, 1963, p. 255). It may be called the historicodevelopmental approach. This approach, which involves the application to history of categories derived from developmental psychology, blends traditional methods of gathering psychological data with the use of data from archival sources. As indicated above, the major archival variable for our work has been the dates of entry of words found in the OED. Use of this variable helps one understand more clearly the way in which the basic, yet diverse, psychological processes involved in the production of imagery (e.g., see Benjafield, $1984,1987)$ and the language of interpersonal behavior (e.g., see Benjafield \& Carson, 1985; Benjafield \& Muckenheim, in press a) are at least partly determined by social and historical forces.

In the present study, we were interested in assembling a set of words representative of those found in the OED, and then getting norms for each word on well-known psychological scales that measure the words' values in terms of familiarity (e.g., see Toglia \& Batig, 1978), imagery (e.g., see Paivio, Yuille, \& Madigan, 1968), concreteness (e.g., see Paivio, Yuille, \& Madigan, 1968), and goodness (e.g., see Brown \& Ure, 1969; Rubin \& Friendly, 1986). Such norms should be useful to researchers interested in sampling very uncommon or unfamiliar words, as well as quite common and familiar ones. They might also be useful to investigators who wish to sample from another database in order to replicate findings obtained from earlier lists. Finally, for the reasons 
noted above, researchers particularly concerned with using a sample that is fairly representative of the range of words in the written language should find the database particularly valuable.

\section{METHOD}

\section{Materials}

Our goal was to obtain data on at least 1,000 words. We accomplished this by initially selecting one word from every 21 st page of the OED, including the supplementary volumes, according to the following selection procedure: Each word was randomly chosen from those on a particular page. A word was excluded, however, if the OED indicated that the word was obsolete or archaic, that it was from a dialect or not naturalized, or that it had no date of entry. Approximately $18 \%$ of the words initially selected were thus excluded. When a word was excluded, another word was randomly chosen from the same page. Occasionally, it was necessary to go on to the next page to find a suitable word. This procedure, which was repeated until we reached the end of the dictionary, yielded a total of 1,046 words. Of these 1,046, 54 were randomly chosen and repeated in the database. This was done to enable reliabilities for the scales to be determined. Thus, the total number of items for which ratings were obtained was 1,100 .

\section{Subjects}

A total of 120 undergraduate student volunteers $(60$ males and 60 females) participated in the study. The total $N$ was divided into four randomly assigned groups of 15 men and 15 women, each of which completed one of the four scales described below. By participating, the subjects fulfilled one of the requirements of the introductory psychology course at Brock University.

\section{Procedure}

All the subjects were run individually, seated in front of a microcomputer terminal. Each subject's data was collected in two sessions of approximately $1 \mathrm{~h}$ each, approximately 2 days apart.

Presentation of words. Each word was entered as a separate record in Reflex (1985), a database program. The design of the record varied, depending on the scale for which the data were being collected. However, regardless of the particular scale, the subject would initially see each word with a seven-point scale below it, anchored by the words defining the scale (e.g., low imagery vs. high imagery). The subject entered a rating for the word on the numeric keypad of the microcomputer, and then, by pressing one key, was shown the next record. The subject continued in this way until having rated items for approximately $1 \mathrm{~h}$. No subject completed all 1,100 ratings in the first session. All returned for the second session, to complete their ratings.
The use of Reflex (1985) made it possible to present the items in a different random order for each subject. Each subject's ratings were separately recorded in Reflex (1985).

Instructions. Except for the instructions for the goodness scale, the printed instructions given to the subjects were adapted from Toglia and Battig (1978), but modified to accommodate the different procedure.

\section{Imagery Scale Instructions}

"Words differ in their capacity to arouse mental images of things or events. Some words arouse a sensory experience, such as a mental picture or sound, very quickly and easily, whereas other words may do so only with difficulty (i.e., after a long delay) or not at all. In this experiment you will rate a list of words as to the ease or difficulty with which they arouse mental images. Any word that in your estimation arouses a mental image (i.e., a mental picture, or sound, or other sensory experience) very quickly and easily should be given a high imagery rating (at the upper end of the numerical scale). Any word that arouses a mental image with difficulty or not at all should be given a low imagery rating (at the lower end of the numerical scale). For example, think of the word 'buffalo.' 'Buffalo' would probably arouse an image relatively easily and would be rated as high imagery; 'relevant' would probably do so with difficulty and be rated as low imagery. Because words tend to make you think of other words as associates, it is important that your ratings not be based on this and that you judge only the ease with which you get a mental image of an object or event in response to each specific word shown.

"Your ratings will be made on a seven-point scale. Feel free to use the entire range of numbers, from 1 to 7 ; at the same time, don't be concerned about how often you use a particular number as long as it is your true judgment.

"After typing in a number rating, press the F8 key. Another word will appear on the screen, and the Rating space will clear, allowing you to type in your rating for this new word. The rating scale itself will remain on the screen for you to refer to.

"You will be rating a total of 1,100 words, approximately half of them today and the other half at your next session. Work fairly quickly, but do not be careless in your ratings. Take breaks if you like. If necessary, refer back to these instructions when rating the words on the screen. If you'd like to know how many words you've rated at any time, just let me know."

After reading the instructions, the subject was told the following by the experimenter.

"Here are four examples of what I would like you to do. What rating would you give to AUTOMOBILE? How about WRITER? What about DEMOCRACY? Finally, please tell me your rating of VAPOR. OK? Any questions?"'

The last three paragraphs of the written instructions and the verbal instructions were common to all ratings, and will not be repeated below. 


\section{Concreteness Scale Instructions}

"Words differ in the extent to which they refer to concrete objects, persons, places, or things that can be seen, heard, felt, smelled, or tasted, as contrasted with abstract concepts that cannot be experienced by our senses. In this experiment you will rate a list of words with respect to their 'concreteness' in terms of sense experience. Any word that refers to objects, materials, or persons would be given a rating at the upper end of the numerical scale. Any word that refers to an abstract concept that cannot be experienced by the senses should be given a rating at the lower end of the numerical scale. For example, think of the word 'carpet,' which can be experienced by our senses and therefore should be rated as highly concrete; the word 'ambiguous' cannot be experienced by the senses as such and therefore should be rated as abstract. Because words tend to make you think of other words as associates, it is important that your ratings not be based on this and that you judge only the concreteness of sense experiences as directly aroused by each specific word shown."

\section{Familiarity Scale Instructions}

"Words differ in their familiarity - that is, how commonly or frequently they have been experienced or how familiar they seem to be. Some words are very familiar, whereas others may be almost totally unfamiliar. In this experiment you will rate a list of words with respect to how familiar or common they are-that is, their familiarity. Any word that appears very common or familiar to you should be given a high familiarity rating (at the upper end of the numerical scale). Any word that you are unfamiliar with, or that is very new to you, would be given a low familiarity rating (at the lower end of the numerical scale). For example, the word 'person' should be very familiar to you and would be rated as highly familiar. A word such as 'amorphous,' on the other hand, is likely to be very unfamiliar to you and therefore should be rated as of low familiarity. Because words also differ in many other ways, such as how many other words they make you think of or how easily they can be mentally imaged, it is important that your ratings not be based on these other characteristics and that you judge only how familiar each specific word shown is to you."

\section{Goodness Scale Instructions}

These instructions were based on Brown and Ure's (1969) goodness scale instructions.

"For an experiment on certain properties of words, we would like you to rate words on the dimension of 'goodness.' A seven-point scale will be used. For each word, decide whether it elicits a feeling of goodness or badness, and how intensely good or bad the feeling is. Type in a number from 1 to 7 accordingly, with lower numbers representing the 'bad' end of the scale, and higher numbers the 'good' end. Because words tend to make you think of other words as associates, it is important that your ratings not be based on this and that you judge only the feeling as directly aroused by the specific word on the screen."

\section{RESULTS AND DISCUSSION}

Mean values for all words for all four scales, as well as the dates of entry, are given in the Appendix. Words that can be found on the word-frequency lists of either Kučera and Francis (1967) or Carroll, Davies, and Richman (1971) are given a score of 1 under the column labeled FREQ; otherwise, they are given a score of 0. This dummy variable was included in order to enable researchers to select words for which frequency data is available. Approximately $48 \%$ of our words can be found in at least one of these two word-frequency lists.

This database is also available on disk for MS-DOS microcomputers. Versions on $5.25^{\prime \prime}$ or $3.5^{\prime \prime}$ disks, in either ASCII or SPSS-PC+ system file formats, are available from the first author at cost.

Descriptive statistics and correlations for all variables are given in Table 1. The skewness and kurtosis values imply that the scale distributions have the following forms. Concreteness and goodness are positively skewed, while familiarity and imagery are negatively skewed, the latter only slightly. All distributions are at least somewhat platykurtic, with familiarity and imagery being the most strikingly so.

Rubin (1980) provides correlational data for date of entry and all the scales for which we also have data, and it is instructive to compare our correlations with his. In Rubin's (1980, Table 1A) data, the correlations between date of entry and all other variables are smaller than ours, although they are all in the same direction. As Rubin $(1980$, p. 747$)$ noted, it is likely that the correlations derived from his data will underestimate the true values because the range of words sampled in his study (and most others) "may not be representative of the total possible range."

\section{Reliability}

Reliability was assessed in two ways. First, the means for the 54 words that were repeated for each variable were

Table 1

Correlations and Descriptive Statistics

for Date-of-Entry (DOE), Concreteness (CONC), Imagery (IMAG), Goodness (GOOD), and Familiarity (FAM)

\begin{tabular}{lrrrrrr}
\hline Variable & DOE & CONC & IMAG & GOOD & FAM & FREQ \\
\hline DOE & 1.00 & & & & & \\
CONC & -.35 & 1.00 & & & & \\
IMAG & -.57 & .64 & 1.00 & & & \\
GOOD & -.32 & .18 & .39 & 1.00 & & \\
FAM & -.59 & .42 & .91 & .45 & 1.00 & \\
FREQ & -.59 & .38 & .64 & .34 & .72 & 1.00 \\
Mean & 1516.21 & 3.81 & 4.03 & 3.90 & 4.59 & .48 \\
SD & 340.51 & 1.16 & 1.70 & .96 & 2.05 & .50 \\
Skewness & -.77 & .85 & -.01 & .41 & -.22 & .10 \\
Kurtosis & -.36 & -.19 & -1.31 & -.12 & -1.48 & -1.99 \\
\hline
\end{tabular}

Note-FREQ is a dummy variable coding whether or not a word is present in either Kučra and Francis (1968) or Carroll and Richman (1971). 
correlated. These correlations were as follows: .99 for imagery; .98 for concreteness; .98 for goodness; and .99 for familiarity.

The second set of reliability estimates can be derived from the 30 complete lists obtained for each scale. These data can be used to calculate Cronbach's alpha, which is a measure of internal consistency based on the average correlation of subjects' ratings. Cronbach's alpha was .97 for imagery; .94 for concreteness; .94 for goodness; and .98 for familiarity.

\section{Comparison to Other Norms}

The following numbers of words were common to both our sample and the following samples: 134 words with the Colorado sample of Toglia and Battig (1978); 33 words with the Toronto sample of Friendly, Franklin, Hoffman, and Rubin (1982); 31 words with the Aberdeen sample of Brown and Ure (1969); and 25 words with the Western Ontario sample of Paivio, Yuille, and Madigan (1968). Although the $N$ s are quite small, a comparison between the Brock University ratings and the others is of interest.

In what follows, matched $t$-tests were used. As Rubin (1980, p. 747$)$ has pointed out, it makes sense to use words as observations rather than subjects because we wish to generalize across words. This requires the assumption that words are independent observations, which is, of course, only approximately true.

The relevant data are given in Table 2. Notice that, in every instance, the Brock University ratings are higher than the corresponding ratings in other samples. These differences are significant $(p<.05)$ for all the scales except concreteness. Only the Colorado University sample is significantly lower in concreteness than the Brock sample $(p<.05)$. The words common to our sample and other samples tend to be words with which subjects are

Table 2

Correlations Between Mean Ratings

for the Brock Word Sample and Mean Ratings for the Colorado, Toronto, Aberdeen, and Western Ontario Word Samples

\begin{tabular}{|c|c|c|c|c|c|c|}
\hline & Correlation & $M$ & $S D$ & $M$ & $S D$ & $N$ \\
\hline & & \multicolumn{2}{|c|}{ Colorado } & \multicolumn{2}{|c|}{ Brock } & \\
\hline Imagery & .87 & $4 . \overline{60}$ & 1.06 & 5.82 & 1.03 & 134 \\
\hline Concreteness & .95 & 4.32 & 1.15 & 4.76 & 1.48 & 134 \\
\hline Familiarity & .82 & 6.14 & .51 & 6.86 & .46 & 134 \\
\hline \multicolumn{7}{|c|}{ Toronto } \\
\hline Imagery & .90 & $4.1 \overline{6}$ & 1.52 & 5.63 & .91 & 33 \\
\hline Concreteness & .86 & 4.19 & 1.48 & 4.25 & 1.33 & 33 \\
\hline \multicolumn{7}{|c|}{ Aberdeen } \\
\hline Concreteness & .80 & 4.58 & 1.16 & 4.83 & 1.42 & 31 \\
\hline Goodness & .89 & 4.62 & 1.08 & 5.08 & 1.04 & 31 \\
\hline \multicolumn{7}{|c|}{ Western } \\
\hline Imagery & .88 & $5.4 \overline{2}$ & 1.11 & 6.23 & .76 & 24 \\
\hline Concreteness & .89 & 5.44 & 1.31 & 5.66 & 1.26 & 24 \\
\hline Goodness & .94 & 4.39 & .95 & 5.05 & 1.21 & 24 \\
\hline
\end{tabular}

Note-The goodness data given for the Western sample are from Rubin and Friendly (1986), who used the same words as did the Western Ontario study. quite familiar. It is likely that the ratings for such relatively familiar words tend to be higher in our sample because the subjects were rating a much larger range of words than is usual in studies of this sort. It is worth repeating Rubin's (1980, p. 747) point that the range of words examined in most psychological experiments is quite small compared to the total possible range.

In spite of these mean differences, the correlations between our ratings and those of other researchers are quite respectable. The lowest correlation is .80 . This outcome implies that the relative positions of words will be fairly stable for any scale across different rating contexts.

\section{Sex differences}

Not only Toglia and Battig (1978) but also Friendly, Franklin, Hoffman, and Rubin (1982, p. 379) have examined sex differences on some scales. The latter investigators concluded that "sex differences may be much more widespread than had been previously suspected." We carried out analyses on each of the scales to see if any sex differences existed in our sample. Again, matched $t$-tests were used.

Mean ratings by men and women for all scales are as follows: men gave significantly higher ratings did than women for concreteness $[M=3.90, S D=1.20$, vs. $M=3.73, S D=1.18 ; t(1045)=10.12, p<.001]$ and for familiarity $[M=4.69, S D=1.99$, vs. $M=4.49$, $S D=2.16 ; t(1045)=10.73, p<.001]$. However, women gave significantly higher ratings for imagery than did men $[M=4.10, S D=1.88$, vs. $M=3.96, S D=$ $1.58 ; t(1045)=6.80, p<.001]$. Men and women did not differ, however, on the goodness scale, where the means are $3.90(S D=.94)$ for men and $3.90(S D=1.04)$ for women. Friendly, Franklin, Hoffman, and Rubin (1982) reported no significant sex differences for imagery and concreteness, which were the only scales they used. It is possible that, once again, the difference in the range of words used in the two studies accounts for the dissimilar results.

For each scale, we also counted the number of items for which the mean ratings of men and women differed by at least .5 . We divided these items into those for which women gave the higher ratings, and those for which men gave the higher ratings. The former class is larger than the latter for imagery ( 302 vs. $182, p<.001$; binomial test), but the reverse is the case for concreteness (111 vs. $272, p<.001)$ and familiarity (86 vs. $265, p<.001)$. No such differences occurred for goodness (148 vs. 151). These results suggest that exploring the types of items for which men and women give markedly different ratings might be a fruitful endeavor. Items for which the female mean was higher than the male one by .5 or greater are marked with the letter $F$ in the Appendix, while those items for which the male mean exceeds the female by at least .5 are marked with $M$.

These differences should not be allowed to obscure the fact that the mean ratings for men and women are very highly correlated. These values are: .88 for goodness; .90 for concreteness; .94 for imagery; and .96 for familiarity. 


\section{REFERENCES}

BENJAFIELD, J. (1983). Some psychological hypotheses concerning the evolution of constructs. British Journal of Psychology, 74, 47-59.

BENJAFIELD, J. (1984). An historicodevelopmental aspect of imagery. Canadian Journal of Behavioural Science, 16, 175-180.

BENJAFTELD, J. (1987a). An historical, social analysis of imagery and concreteness. British Journal of Social Psychology, 26, 155-164.

BENJAFIELD, J. (1987b). An historicodevelopmental analysis of the frequency with which construct poles are used. Canadian Journal of Behavioural Science, 19, 332-341.

Benjafield, J., \& Carson, E. (1985). An historiçodevelopmental analysis of the circumplex model of trait descriptive terms. Canadian Journal of Behavioural Science, 17, 339-345.

Benjafield, J., \& CARSON, E. (1986). The image arousing potential of proverbs as a function of source and mode. British Joumal of Social Psychology, 25, 51-56.

Benjafield, J., \& MuCKenheim, R. (in press a). A further historicodevelopmental study of the interpersonal circumplex. Canadian Joumal of Behavioural Science.

Benjafield, J. \& Muckenheim, R. (in press b). An historicodevelopmental analysis of the Regressive Imagery Dictionary. Empirical Studies of the Arts.

Brown, W. P., \& URE, D. M. J. (1969). Five rated characteristics of 650 word association stimuli. British Journal of Psychology, 60, 232-249

Carroll, J. B., Davies, P., \& Richman, B. (1971). Word frequency book. Boston: Houghton Mifflin.

Flavel., J. H. (1963). The developmental psychology of Jean Piaget. Princeton, NJ: Van Nostrand.

Friendly, M., Frankun, P. E., Hoffman, D., \& Rubin, D. C. (1982). The Toronto Word Pool: Norms for imagery, concreteness, ortho- graphic variables, and grammatical usage for 1,080 words. Behavior Research Methods \& Instrumentation, 14, 375-399.

GilHOOLY, K. J. \& LOGIE, R. H. (1980). Age-of-acquisition, imagery, concreteness, familiarity, and ambiguity measures for 1,944 words. Behavior Research Methods \& Instrumentation, 12, 395-427.

KuČERA, H., \& Francis, W. H. (1967). Computational analysis of present-day American E-nglish. Providence, RI: Brown University Press.

Olson, D., Astington, J. W. (1986). Children's acquisition of metalinguistic and metacognitive verbs. In W. Demopoulos \& A. Marras (Eds.), Language learning and concept acquisition: Foundational issues. Norwood, NJ: Ablex.

The Oxford English Dictionary. (1933-1986). Oxford: Oxford University Press.

Paivio, A., Yuille, J., \& Madigan, S. A. (1968). Concreteness, imagery, and meaningfulness values for 925 nouns. Joumal of Experimental Psychology Monographs, 76(1, Pt. 2).

Piaget, J. (1950). Introduction a l'épistémologie génétique. Paris: Presses Universitaires de France.

ReFLeX [Computer program]. (1985). Scott's Valley, CA: Borland International.

Rubin, D. C. (1980). 51 properties of 125 words: A unit analysis of verbal behavior. Journal of Verbal Learning \& Verbal Behavior, 19, 736-755.

Rubin, D. C., \& Friendly, M. (1986). Predicting which words get recalled: Measures of free recall, availability, goodness, emotionality, and pronunciability for 925 nouns. Memory \& Cognition, 14, 79-94.

Toglia, M. P. \& Battig, W. F. (1978). Handbook of semantic word norms. Hillsdale, $\mathrm{NJ}$ : Erlbaum.

WILLIAMS, R. (1983). Keywords: A vocabulary of culture and society. London: Fontana.

\section{APPENDIX}

Date-of-Entry (DOE) and Norms for Concreteness (CONC), Imagery (IMAG), Goodness (GOOD), and Familiarity (FAM).

FREQ is a dummy variable coding the presence (1) or absence ( 0 ) of a word in either the Kucera and Francis (1968) or the Carroll and Richman (1971) word-frequency lists.

\begin{tabular}{|c|c|c|c|c|c|c|c|c|c|c|}
\hline WORD & DOE & CONC & & IMAG & & GOOD & & FAM & & FREQ \\
\hline$A$ & 1000 & 2.11 & & 3.20 & F & 4.63 & & 6.40 & & 1 \\
\hline a-quarter & 1849 & 4.10 & $\mathbf{M}$ & 4.97 & & 4.37 & . & 3.74 & . & 0 \\
\hline ablaut & 1849 & 2.33 & & 1.40 & $\mathbf{M}$ & 3.27 & & 1.37 & & 0 \\
\hline absenteeship & 1778 & 2.83 & & 3.27 & $\mathrm{~F}$ & 2.53 & & 3.94 & $\mathbf{F}$ & $\mathbf{0}$ \\
\hline abthainry & 1872 & 2.40 & & 1.43 & $\mathbf{M}$ & 3.47 & $\mathbf{M}$ & 1.34 & . & 0 \\
\hline account & 1260 & 4.67 & $\mathbf{M}$ & 5.07 & & 4.37 & . & 6.97 & & 1 \\
\hline acquirable & 1646 & 2.73 & & 3.70 & & 5.44 & . & 6.00 & & 1 \\
\hline addressor & 1691 & 4.43 & . & 3.77 & $\mathbf{F}$ & 4.07 & & 4.90 & $\mathbf{M}$ & 0 \\
\hline adoptee & 1892 & 4.33 & . & 5.34 & $\mathbf{F}$ & 4.63 & $\mathbf{F}$ & 5.90 & . & 0 \\
\hline adrogated & 1875 & 2.43 & & 1.94 & & 3.23 & 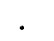 & 1.93 & & 0 \\
\hline aestivation & 1625 & 2.57 & $\mathbf{M}$ & 1.37 & $\mathbf{M}$ & 3.57 & . & 1.67 & $\mathbf{M}$ & 0 \\
\hline aftercoming & 1382 & 2.27 & & 3.33 & $F$ & 4.10 & & 5.04 & . & 0 \\
\hline agreement & 1398 & 4.00 & $\mathbf{M}$ & 4.77 & & 5.64 & . & 6.93 & . & 1 \\
\hline air & 822 & 5.47 & $\mathbf{M}$ & 5.97 & $\mathbf{M}$ & 6.20 & & 6.84 & . & 1 \\
\hline airway & 1851 & 5.03 & & 5.73 & & 5.34 & $\mathbf{F}$ & 6.67 & . & 0 \\
\hline alcyon & 1868 & 3.33 & $\mathbf{M}$ & 1.57 & $\mathbf{M}$ & 3.13 & . & 1.50 & & 0 \\
\hline allegorizing & 1579 & 2.37 & & 2.73 & & 3.10 & & 3.17 & $\mathbf{M}$ & $\mathbf{0}$ \\
\hline alpigene & 1847 & 3.20 & $\mathbf{M}$ & 1.47 & $\mathbf{M}$ & 3.40 & & 1.44 & . & 0 \\
\hline alternating & 1837 & 2.97 & & 4.73 & & 4.80 & $\mathbf{F}$ & 6.94 & & 1 \\
\hline ambulating & 1786 & 2.80 & $\mathbf{M}$ & 2.40 & $\mathbf{M}$ & 3.83 & & 3.13 & $\mathbf{M}$ & 0 \\
\hline ampulliform & 1870 & 3.33 & & 1.50 & & 3.23 & $\mathbf{M}$ & 1.37 & & 0 \\
\hline anconoid & 1819 & 2.97 & $\mathbf{M}$ & 1.43 & $\mathbf{M}$ & 3.30 & . & 1.30 & & 0 \\
\hline angon & 1875 & 3.17 & 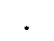 & 1.50 & $\mathbf{M}$ & 3.04 & . & 1.74 & & 0 \\
\hline anisotropic & 1879 & 2.80 & & 1.70 & & 3.37 & & 1.53 & & 0 \\
\hline ant-fly & 1653 & 5.57 & & 5.03 & & 2.60 & & 3.50 & & 0 \\
\hline aortic & 1833 & 3.37 & & 3.37 & & 4.10 & $\mathrm{~F}$ & 4.06 & $\mathbf{M}$ & 0 \\
\hline
\end{tabular}


APPENDIX (Continued)

\begin{tabular}{|c|c|c|c|c|c|c|c|c|c|c|}
\hline WORD & DOE & CONC & & IMAG & & GOOD & & FAM & & FREQ \\
\hline apparentation & 1934 & 2.77 & . & 2.40 & ${ }^{\circ}$ & 4.10 & $\mathbf{M}$ & 2.60 & $\mathbf{F}$ & 0 \\
\hline appeaser & 1533 & 3.97 & . & 2.84 & & 3.67 & $\mathbf{M}$ & 4.33 & $\mathbf{M}$ & $\mathbf{0}$ \\
\hline arctoid & 1869 & 3.13 & . & 1.80 & $\mathbf{M}$ & 3.44 & . & 1.50 & $\mathbf{M}$ & 0 \\
\hline argotic & 1863 & 2.67 & 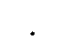 & 1.77 & . & 3.40 & . & 1.73 & . & 0 \\
\hline arrogating & 1574 & 2.47 & & 2.43 & . & 2.97 & $\mathbf{M}$ & 3.27 & F & 1 \\
\hline ascititious & 1628 & 2.37 & $\mathbf{M}$ & 1.60 & . & 3.03 & . & 1.84 & 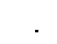 & 0 \\
\hline ash & 700 & 6.33 & . & 6.73 & & 2.87 & $\mathbf{M}$ & 6.94 & . & 1 \\
\hline assentiveness & 1876 & 2.27 & . & 3.00 & $\mathbf{M}$ & 4.33 & $\mathrm{~F}$ & 3.37 & . & 0 \\
\hline astronomically & 1649 & 2.60 & . & 4.77 & . & 5.27 & . & 5.70 & $\mathbf{M}$ & 1 \\
\hline attempting & 1556 & 2.63 & F & 4.50 & $\mathrm{~F}$ & 5.27 & . & 6.64 & . & 1 \\
\hline auspiciousness & 1649 & 2.20 & & 2.73 & 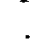 & 4.00 & . & 4.00 & $\mathbf{M}$ & 0 \\
\hline Aussie & 1917 & 5.37 & $\mathbf{M}$ & 4.10 & $\mathbf{M}$ & 4.94 & $\mathbf{M}$ & 5.04 & $\mathbf{M}$ & 0 \\
\hline avow & 1220 & 2.57 & . & 2.04 & $\mathbf{M}$ & 3.87 & $\mathrm{~F}$ & 3.24 & $\mathbf{M}$ & 0 \\
\hline back & 885 & 5.93 & . & 6.27 & $\mathbf{F}$ & 4.37 & . & 7.00 & . & 1 \\
\hline backband & 1523 & 4.17 & . & 3.83 & $\mathbf{M}$ & 3.73 & $\mathrm{~F}$ & 3.70 & . & 0 \\
\hline balance & 1275 & 4.40 & . & 5.77 & F & 5.57 & . & 6.94 & . & 1 \\
\hline balk & 885 & 3.90 & . & 4.50 & . & 3.37 & $\mathbf{M}$ & 5.10 & $\mathbf{M}$ & 1 \\
\hline banak & 1921 & 3.60 & $\mathrm{~F}$ & 1.77 & $\mathbf{M}$ & 3.40 & . & 1.70 & $\mathbf{M}$ & 0 \\
\hline banish & 1375 & 3.37 & . & 5.40 & . & 2.63 & . & 6.54 & . & 1 \\
\hline barking & 1300 & 5.47 & . & 6.50 & . & 3.57 & $\mathbf{F}$ & 6.90 & . & 1 \\
\hline bassoonist & 1865 & 5.77 & . & 4.30 & . & 4.13 & . & 3.93 & . & 1 \\
\hline batement & 1445 & 3.30 & . & 2.13 & . & 3.20 & . & 2.54 & $\mathbf{F}$ & 0 \\
\hline bdellometer & 1839 & 4.63 & . & 1.83 & . & 3.07 & . & 1.27 & $\mathbf{M}$ & 0 \\
\hline bearing & 1250 & 3.97 & $\mathbf{M}$ & 5.17 & $\mathrm{~F}$ & 4.97 & . & 6.93 & . & 1 \\
\hline bee-eater & 1668 & 5.60 & . & 5.27 & . & 2.63 & . & 3.84 & $\mathbf{M}$ & 0 \\
\hline being & 1300 & 3.03 & $\mathbf{M}$ & 4.33 & $\dot{F}$ & 5.17 & . & 7.00 & . & 1 \\
\hline bekiss & 1587 & 2.83 & . & 2.44 & . & 4.07 & . & 1.94 & . & 0 \\
\hline beneath & 854 & 3.37 & . & 5.67 & . & 3.57 & . & 7.00 & . & 1 \\
\hline besmeared & 1592 & 2.53 & $\mathbf{F}$ & 2.94 & $\mathbf{F}$ & 2.53 & $\mathbf{M}$ & 3.07 & . & 0 \\
\hline beyond & 1000 & 2.67 & $\mathbf{M}$ & 5.47 & . & 4.80 & . & 6.93 & . & 1 \\
\hline biblioclast & 1880 & 3.20 & . & 1.83 & . & 3.80 & $\mathrm{~F}$ & 2.23 & . & 0 \\
\hline bilabial & 1862 & 2.97 & . & 1.83 & . & 3.20 & $\mathbf{M}$ & 1.90 & $\mathbf{M}$ & 0 \\
\hline bill & 1000 & 6.00 & . & 6.40 & $\mathbf{F}$ & 3.30 & $\mathbf{F}$ & 6.97 & . & 1 \\
\hline bite & 1000 & 5.83 & . & 6.27 & $\mathbf{F}$ & 2.80 & . & 7.00 & . & 1 \\
\hline blackbutt & 1801 & 4.27 & . & 4.10 & . & 2.60 & . & 2.83 & . & 0 \\
\hline blasphemously & 1531 & 2.30 & . & 4.23 & . & 2.37 & $\mathrm{~F}$ & 5.17 & . & 0 \\
\hline bloat & 1300 & 4.30 & . & 6.14 & $\mathbf{F}$ & 2.13 & . & 6.37 & . & 1 \\
\hline blow & 1000 & 4.70 & . & 6.43 & $\mathbf{F}$ & 3.93 & . & 6.90 & . & 1 \\
\hline bluet & 1300 & 3.00 & . & 2.47 & 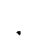 & 3.34 & . & 2.20 & $\mathbf{M}$ & 0 \\
\hline boardy & 1893 & 2.90 & . & 3.30 & $\mathbf{F}$ & 3.47 & . & 3.10 & $\mathbf{M}$ & 0 \\
\hline boglet & 1869 & 3.30 & . & 2.20 & . & 3.34 & $\mathbf{F}$ & 1.70 & $\mathbf{M}$ & 0 \\
\hline bonelessness & 1885 & 3.43 & . & 5.67 & . & 2.60 & . & 4.97 & $\mathrm{~F}$ & 0 \\
\hline bonxie & 1802 & 2.90 & . & 1.60 & $\mathbf{M}$ & 3.64 & . & 1.44 & . & 0 \\
\hline boss & 1300 & 5.37 & $\mathbf{M}$ & 6.63 & $\mathbf{F}$ & 4.27 & . & 7.00 & . & 1 \\
\hline bow & 893 & 6.53 & . & 6.47 & . & 4.13 & $\mathbf{F}$ & 6.97 & . & 1 \\
\hline boxer & 1742 & 6.57 & . & 6.77 & . & 3.54 & $\mathbf{M}$ & 6.90 & . & 1 \\
\hline bramantip & 1870 & 2.80 & . & 1.37 & & 3.50 & . & 1.23 & . & $\mathbf{0}$ \\
\hline break & 851 & 4.50 & . & 6.34 & $\mathrm{~F}$ & 3.03 & . & 6.97 & . & 1 \\
\hline bribing & 1542 & 3.50 & . & 5.50 & . & 2.10 & $\mathbf{M}$ & 6.47 & . & 0 \\
\hline brickbat & 1563 & 4.47 & $\mathbf{M}$ & 3.17 & . & 3.57 & . & 2.10 & $\mathbf{M}$ & 0 \\
\hline britoness & 1591 & 3.50 & . & 1.97 & . & 3.37 & $\mathbf{M}$ & 1.63 & $\mathbf{M}$ & 0 \\
\hline broadside & 1575 & 4.33 & . & 5.80 & . & 3.53 & $\mathbf{M}$ & 6.44 & $\mathbf{M}$ & 1 \\
\hline browbeaten & 1747 & 2.90 & . & 4.01 & $\mathbf{F}$ & 2.47 & . & 5.53 & $\mathbf{M}$ & 1 \\
\hline budgeter & 1603 & 4.30 & $\mathbf{F}$ & 4.54 & $F$ & 4.33 & $\mathbf{M}$ & 5.07 & . & 0 \\
\hline bully & 1538 & 4.97 & $\mathbf{M}$ & 6.40 & . & 2.00 & . & 6.77 & . & 1 \\
\hline bunchiness & 1594 & 3.00 & . & 4.17 & $\mathrm{~F}$ & 3.27 & $\mathrm{~F}$ & 4.20 & . & 0 \\
\hline burst & 1000 & 3.73 & . & 6.07 & . & 3.47 & $\mathbf{F}$ & 6.94 & . & 1 \\
\hline bushman & 1785 & 6.00 & $\mathbf{M}$ & 5.93 & $\mathrm{~F}$ & 3.83 & $\mathrm{M}$ & 5.67 & $\mathbf{M}$ & 1 \\
\hline butter-fingers & 1837 & 3.70 & $\mathbf{F}$ & 5.77 & $\mathbf{F}$ & 3.00 & $\mathbf{F}$ & 6.47 & $\mathrm{~F}$ & 0 \\
\hline buzzer & 1602 & 6.13 & $\mathbf{M}$ & 6.47 & . & 3.10 & . & 6.64 & . & 1 \\
\hline by-walk & 1549 & 3.67 & . & 3.63 & $\mathbf{F}$ & 4.37 & $\mathbf{F}$ & 3.37 & . & 0 \\
\hline
\end{tabular}


APPENDIX (Continued)

\begin{tabular}{|c|c|c|c|c|c|c|c|c|c|c|}
\hline WORD & DOE & CONC & & IMAG & & GOOD & & FAM & & REQ \\
\hline cajolement & 1816 & 2.77 & & 2.30 & . & 3.33 & & 2.50 & & 0 \\
\hline calorocity & 1836 & 2.77 & & 2.40 & . & 3.40 & & 1.73 & & 0 \\
\hline candescence & 1880 & 3.37 & & 2.24 & & 4.34 & $\mathbf{F}$ & 3.07 & & $\mathbf{0}$ \\
\hline cankery & 1398 & 3.20 & $\mathbf{M}$ & 3.13 & $\mathbf{M}$ & 2.63 & $\mathbf{M}$ & 3.14 & . & 0 \\
\hline cant-spar & 1611 & 3.63 & $\mathbf{M}$ & 1.53 & $\mathbf{M}$ & 3.17 & . & 1.74 & . & 0 \\
\hline carap & 1865 & 3.33 & $\mathbf{M}$ & 1.63 & & 3.07 & & 1.34 & & 0 \\
\hline caritative & 1884 & 2.73 & & 1.90 & $\mathbf{M}$ & 3.73 & $\mathbf{M}$ & 2.27 & $\mathbf{M}$ & 0 \\
\hline caroling & 1300 & 4.47 & F & 5.93 & $\mathbf{F}$ & 5.83 & F & 6.27 & $\mathrm{~F}$ & 1 \\
\hline carver & 1380 & 5.27 & & 5.64 & $\mathrm{~F}$ & 4.30 & & 5.77 & & 1 \\
\hline case-hardened & 1691 & 3.60 & $\mathbf{M}$ & 3.20 & $\mathbf{M}$ & 3.40 & $\mathbf{M}$ & 3.87 & $\mathbf{M}$ & 1 \\
\hline cat & 800 & 6.87 & . & 6.74 & $\mathrm{~F}$ & 5.27 & $\mathbf{F}$ & 7.00 & & 1 \\
\hline cattle & 1205 & 6.43 & & 6.77 & . & 4.73 & . & 6.94 & & 1 \\
\hline celation & 1567 & 2.87 & & 1.80 & . & 3.97 & 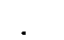 & 1.64 & & 0 \\
\hline centisecond & 1950 & 3.07 & $F$ & 3.13 & . & 3.47 & $\mathbf{M}$ & 3.00 & $\mathbf{M}$ & 0 \\
\hline cerement & 1602 & 3.33 & $\mathbf{M}$ & 2.10 & . & 3.43 & . & 1.97 & & 0 \\
\hline chalder & 1300 & 3.00 & & 1.90 & & 3.23 & & 2.00 & $\mathbf{M}$ & 0 \\
\hline chap & 1225 & 5.37 & $\mathrm{~F}$ & 5.70 & $\mathrm{~F}$ & 3.83 & $\mathbf{M}$ & 6.57 & . & 1 \\
\hline charging & 1568 & 3.40 & . & 5.93 & . & 3.23 & $\mathbf{M}$ & 6.83 & . & 1 \\
\hline chartaceous & 1655 & 2.43 & . & 2.17 & & 3.37 & . & 2.04 & . & 0 \\
\hline check & 1314 & 4.30 & . & 5.93 & $\mathrm{~F}$ & 5.00 & . & 6.90 & . & 1 \\
\hline cheerfulness & 1535 & 3.63 & . & 5.80 & & 6.67 & . & 6.93 & . & 1 \\
\hline chick & 1320 & 6.27 & & 6.34 & $\mathrm{~F}$ & 5.04 & . & 6.97 & . & 1 \\
\hline chipping & 1440 & 4.13 & $\mathbf{M}$ & 6.14 & & 3.50 & . & 6.10 & . & 1 \\
\hline choking & 1440 & 5.10 & $\mathrm{~F}$ & 6.53 & $\mathrm{~F}$ & 1.47 & . & 6.87 & . & 1 \\
\hline chop & 1362 & 5.40 & . & 6.53 & & 3.47 & . & 7.00 & . & 1 \\
\hline chthonography & 1881 & 3.17 & & 1.37 & $\mathbf{M}$ & 3.44 & . & 1.40 & . & 0 \\
\hline cinnyl & 1879 & 3.00 & & 1.47 & $\mathbf{M}$ & 2.83 & & 1.53 & & 0 \\
\hline circumflex & 1565 & 3.37 & $\mathrm{~F}$ & 3.70 & $\mathrm{~F}$ & 4.40 & $\mathrm{~F}$ & 4.30 & & 0 \\
\hline cirrate & 1826 & 3.03 & & 1.90 & & 2.90 & & 1.63 & $\mathbf{M}$ & 0 \\
\hline clamming & 1636 & 3.10 & & 4.00 & & 3.47 & & 4.70 & . & 1 \\
\hline claret & 1396 & 4.43 & & 2.47 & $\mathbf{F}$ & 4.57 & $\mathrm{~F}$ & 3.03 & . & 1 \\
\hline clearing & 1380 & 4.87 & $\mathbf{M}$ & 5.63 & $\mathrm{~F}$ & 5.13 & & 6.90 & & 1 \\
\hline clink & 1386 & 4.73 & F & 5.70 & $\mathrm{~F}$ & 3.93 & $\mathrm{~F}$ & 5.40 & $\mathbf{M}$ & 1 \\
\hline clothes-horse & 1806 & 5.00 & $\mathbf{M}$ & 4.97 & $\mathrm{~F}$ & 4.20 & . & 3.93 & $\mathbf{F}$ & 0 \\
\hline clotting & 1483 & 5.00 & . & 6.07 & $\mathrm{~F}$ & 3.50 & . & 6.50 & & 1 \\
\hline coachman & 1579 & 6.17 & . & 5.53 & $\mathrm{~F}$ & 4.60 & 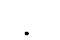 & 5.70 & & 1 \\
\hline cock & 897 & 6.33 & $\mathbf{M}$ & 5.94 & $\mathrm{~F}$ & 3.37 & & 6.84 & & 1 \\
\hline coercive & 1600 & 2.80 & $\mathbf{M}$ & 2.53 & $\mathbf{M}$ & 2.87 & $\mathbf{M}$ & 5.34 & $\mathbf{M}$ & 1 \\
\hline cold & 950 & 5.57 & . & 6.43 & . & 2.97 & $\mathbf{M}$ & 7.00 & & 1 \\
\hline Coleridgian & 1834 & 3.70 & $\mathbf{M}$ & 1.47 & $\mathbf{M}$ & 3.60 & . & 1.40 & $\mathbf{M}$ & 0 \\
\hline colloquize & 1823 & 2.50 & . & 2.34 & & 3.77 & . & 3.13 & $\mathbf{M}$ & 0 \\
\hline come & 825 & 3.00 & & 5.07 & $\mathbf{M}$ & 5.13 & . & 7.00 & . & 1 \\
\hline commenced & 1588 & 2.90 & $\mathbf{M}$ & 4.50 & . & 4.93 & . & 6.60 & . & 1 \\
\hline commonness & 1530 & 2.50 & . & 3.70 & 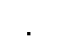 & 4.00 & . & 5.63 & & 1 \\
\hline companionate & 1657 & 3.20 & . & 4.40 & 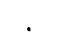 & 5.84 & $\mathrm{~F}$ & 4.64 & $\mathrm{~F}$ & $\mathbf{0}$ \\
\hline comparatist & 1933 & 4.17 & . & 2.63 & & 3.70 & & 3.64 & & $\mathbf{0}$ \\
\hline compasser & 1494 & 4.30 & & 3.14 & F & 4.10 & & 3.53 & $\mathrm{~F}$ & 0 \\
\hline composedness & 1611 & 2.87 & $\mathbf{M}$ & 3.40 & $\mathrm{~F}$ & 4.53 & & 4.73 & & 0 \\
\hline conceitless & 1591 & 2.60 & $\mathrm{~F}$ & 3.57 & $\mathrm{~F}$ & 4.07 & $\mathrm{~F}$ & 4.20 & & 0 \\
\hline concretionary & 1830 & 2.80 & . & 2.17 & $\mathbf{M}$ & 4.03 & . & 2.44 & & 0 \\
\hline confederated & 1605 & 2.87 & & 3.26 & . & 4.17 & & 5.80 & $\mathbf{M}$ & 0 \\
\hline congener & 1730 & 3.13 & $\mathrm{~F}$ & 2.23 & . & 3.73 & $\mathbf{M}$ & 2.04 & & 0 \\
\hline connivence & 1596 & 2.77 & . & 2.43 & & 3.77 & $\mathbf{M}$ & 2.47 & $\mathbf{M}$ & 0 \\
\hline consigning & 1642 & 2.40 & . & 3.10 & $\dot{\mathrm{F}}$ & 4.03 & $\mathbf{M}$ & 4.63 & & 0 \\
\hline consortium & 1829 & 3.23 & & 2.40 & $\mathbf{M}$ & 3.70 & . & 3.44 & $\mathbf{M}$ & 1 \\
\hline consuetudinary & 1494 & 2.50 & $\mathrm{~F}$ & 1.47 & & 2.87 & & 1.54 & & 0 \\
\hline continence & 1340 & 2.63 & $\mathbf{M}$ & 2.97 & $\mathrm{~F}$ & 3.74 & . & 4.00 & $\mathbf{M}$ & 1 \\
\hline contributable & 1611 & 2.57 & . & 3.30 & & 4.70 & . & 5.20 & $\mathrm{~F}$ & 0 \\
\hline convertibility & 1734 & 2.90 & & 3.70 & $\mathrm{~F}$ & 5.24 & . & 5.27 & $F$ & 1 \\
\hline $\operatorname{coot}$ & 1300 & 4.03 & $\mathbf{M}$ & 3.27 & . & 2.64 & & 3.27 & $\mathbf{M}$ & 0 \\
\hline cordelle & 1792 & 3.17 & . & 1.77 & $\mathbf{F}$ & 3.80 & $\mathrm{~F}$ & 1.83 & $\mathbf{M}$ & 0 \\
\hline
\end{tabular}


APPENDIX (Continued)

\begin{tabular}{|c|c|c|c|c|c|c|c|c|c|c|}
\hline WORD & DOE & CONC & & IMAG & & GOOD & & FAM & & FREQ \\
\hline cordwaining & 1758 & 2.50 & & 1.93 & & 3.40 & & 1.60 & $\mathbf{M}$ & 0 \\
\hline coronium & 1890 & 3.93 & & 2.37 & $\mathbf{M}$ & 3.80 & . & 2.34 & & 0 \\
\hline coryniform & 1877 & 3.13 & $\mathbf{M}$ & 1.60 & $\mathbf{M}$ & 3.47 & . & 1.50 & $\mathbf{M}$ & 0 \\
\hline coughing & 1398 & 5.20 & . & 6.70 & & 2.07 & & 6.97 & & 1 \\
\hline counterpoint & 1423 & 3.77 & . & 4.40 & $\mathbf{M}$ & 4.00 & . & 5.97 & $\mathbf{M}$ & 1 \\
\hline county & 1217 & 5.50 & . & 6.13 & . & 5.00 & & 6.77 & . & 1 \\
\hline coupon & 1822 & 6.53 & . & 6.30 & . & 4.40 & $\mathbf{M}$ & 6.87 & . & 1 \\
\hline court & 1154 & 5.90 & & 6.57 & & 4.20 & & 7.00 & & 1 \\
\hline cowe & 1785 & 3.33 & $\mathbf{M}$ & 2.20 & $\mathbf{M}$ & 3.26 & $\mathbf{M}$ & 1.94 & $\mathbf{M}$ & 0 \\
\hline cramp & 1374 & 5.90 & . & 6.17 & & 1.90 & & 6.80 & & 1 \\
\hline credit & 1541 & 4.07 & . & 5.20 & & 5.47 & $\mathbf{M}$ & 6.97 & & 1 \\
\hline cretin & 1779 & 4.40 & . & 2.90 & $\mathbf{M}$ & 3.10 & & 3.70 & $\mathbf{M}$ & 1 \\
\hline cringle & 1627 & 3.67 & . & 3.93 & . & 4.13 & $\mathbf{F}$ & 3.57 & . & 0 \\
\hline crossbill & 1672 & 4.23 & . & 3.04 & . & 3.37 & & 3.60 & . & 1 \\
\hline crumble & 1420 & 4.67 & . & 6.30 & & 2.57 & & 6.87 & . & 1 \\
\hline cube & 1398 & 6.27 & . & 6.67 & . & 4.40 & F & 7.00 & & 1 \\
\hline cudgelling & 1606 & 3.20 & . & 1.84 & . & 3.33 & & 1.74 & $\mathbf{M}$ & 0 \\
\hline curatory & 1560 & 3.10 & . & 2.47 & & 3.70 & $\mathbf{M}$ & 2.44 & $\mathbf{M}$ & 0 \\
\hline cusk & 1624 & 3.73 & . & 1.70 & $\mathbf{M}$ & 3.03 & . & 1.74 & . & 0 \\
\hline cylindered & 1899 & 3.77 & . & 5.03 & $\mathbf{F}$ & 4.13 & . & 4.67 & . & 0 \\
\hline cymar & 1641 & 3.57 & . & 1.33 & & 3.27 & 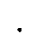 & 1.44 & . & 0 \\
\hline cymbidium & 1815 & 3.40 & . & 1.47 & $\mathbf{M}$ & 3.27 & . & 1.60 & & 0 \\
\hline damask & 1250 & 3.43 & . & 2.30 & F & 3.80 & . & 2.30 & $\mathrm{~F}$ & 1 \\
\hline darkle & 1800 & 3.07 & . & 1.80 & & 3.43 & . & 2.03 & . & 0 \\
\hline dea & 1821 & 2.57 & . & 1.37 & $\mathbf{M}$ & 3.10 & . & 1.67 & & 0 \\
\hline deaconhood & 1382 & 3.67 & & 3.63 & . & 4.10 & . & 3.37 & $\mathbf{M}$ & 0 \\
\hline debating & 1548 & 3.33 & $\mathbf{M}$ & 5.34 & . & 4.00 & . & 6.74 & & 1 \\
\hline declaratorily & 1588 & 2.57 & . & 2.60 & . & 3.80 & . & 3.10 & $\mathbf{M}$ & 0 \\
\hline deep & 854 & 5.07 & . & 6.50 & . & 4.17 & . & 6.97 & & 1 \\
\hline deform & 1382 & 3.80 & . & 5.67 & & 1.87 & . & 6.44 & & 1 \\
\hline degrade & 1325 & 3.07 & . & 4.73 & & 1.93 & . & 6.63 & & 1 \\
\hline deligated & 1840 & 2.60 & . & 4.10 & $\mathrm{~F}$ & 4.47 & . & 5.90 & & 0 \\
\hline demogorgon & 1590 & 3.60 & & 1.97 & $\mathbf{M}$ & 3.23 & & 1.67 & $\mathbf{M}$ & 0 \\
\hline depart & 1225 & 3.93 & & 5.80 & $\mathrm{~F}$ & 3.53 & $\mathbf{M}$ & 6.97 & . & 1 \\
\hline derange & 1776 & 3.07 & $\mathbf{M}$ & 4.50 & . & 1.93 & . & 5.90 & & 0 \\
\hline desire & 1230 & 2.83 & $\mathrm{~F}$ & 5.30 & & 5.80 & . & 7.00 & & 1 \\
\hline determinacy & 1873 & 2.33 & & 3.17 & $\mathrm{~F}$ & 4.84 & & 4.53 & & 0 \\
\hline determinant & 1449 & 3.20 & $\mathbf{M}$ & 3.67 & & 4.40 & & 6.03 & & 1 \\
\hline devastatingly & 1905 & 2.60 & & 4.90 & $\mathbf{M}$ & 2.33 & $\mathbf{M}$ & 5.84 & & 1 \\
\hline devilry & 1375 & 2.47 & & 3.40 & & 2.90 & & 3.37 & & 0 \\
\hline dial & 1430 & 5.87 & $\mathbf{M}$ & 6.34 & $\mathrm{~F}$ & 4.90 & . & 6.97 & & 1 \\
\hline dictatory & 1533 & 3.23 & $\mathbf{M}$ & 4.37 & & 2.97 & & 4.40 & & 0 \\
\hline digestive & 1386 & 3.80 & $\mathbf{M}$ & 5.67 & . & 4.80 & F & 6.90 & & 1 \\
\hline dimorphic & 1859 & 3.10 & & 1.67 & & 3.50 & . & 3.67 & $\mathrm{~F}$ & 0 \\
\hline dinah & 1898 & 3.33 & $\mathbf{M}$ & 2.60 & & 3.67 & & 3.57 & $\mathbf{M}$ & 0 \\
\hline directory & 1450 & 6.03 & $\mathbf{M}$ & 6.13 & $F$ & 4.87 & & 6.87 & . & 1 \\
\hline dischargeable & 1781 & 2.90 & $\mathbf{M}$ & 4.07 & & 3.70 & & 5.87 & & 0 \\
\hline discretionarily & 1683 & 2.21 & . & 3.50 & F & 4.20 & & 4.43 & $\mathrm{~F}$ & 0 \\
\hline dishing & 1669 & 3.30 & & 4.77 & $\mathrm{~F}$ & 4.00 & $\mathbf{F}$ & 5.74 & $\mathrm{~F}$ & 0 \\
\hline disparition & 1594 & 2.53 & & 2.34 & & 3.03 & $\mathbf{M}$ & 3.40 & $\mathrm{~F}$ & 0 \\
\hline dispute & 1225 & 3.70 & & 4.67 & & 2.67 & $\mathbf{M}$ & 6.77 & & 1 \\
\hline distasted & 1651 & 3.07 & F & 4.00 & $\mathrm{~F}$ & 2.74 & 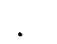 & 4.20 & $\mathbf{F}$ & 0 \\
\hline disyllabify & 1846 & 2.60 & $\mathrm{~F}$ & 1.60 & & 2.80 & & 2.17 & $F$ & 0 \\
\hline diterpene & 1902 & 3.00 & & 1.40 & $\mathbf{M}$ & 3.23 & $\mathbf{M}$ & 1.30 & & 0 \\
\hline $\operatorname{dizz}$ & 1632 & 2.17 & & 3.10 & $\mathbf{M}$ & 3.26 & 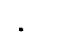 & 2.97 & $F$ & 0 \\
\hline dog-head & 1607 & 5.17 & . & 5.70 & $\mathbf{M}$ & 3.10 & & 4.00 & . & 0 \\
\hline dor & 700 & 2.87 & . & 2.53 & & 3.74 & $\mathbf{M}$ & 1.80 & & 0 \\
\hline dot & 1000 & 6.30 & & 6.40 & $\mathrm{~F}$ & 4.56 & $F$ & 7.00 & & 1 \\
\hline down & 971 & 4.23 & & 6.10 & & 3.10 & . & 7.00 & & 1 \\
\hline drag & 1388 & 4.37 & $\mathrm{~F}$ & 6.27 & & 2.60 & & 6.97 & & 1 \\
\hline draw & 897 & 4.83 & $\mathrm{~F}$ & 6.33 & $\mathbf{F}$ & 5.44 & & 6.97 & & 1 \\
\hline
\end{tabular}


APPENDIX (Continued)

\begin{tabular}{|c|c|c|c|c|c|c|c|c|c|c|}
\hline WORD & DOE & CONC & & IMAG & & GOOD & & FAM & & REQ \\
\hline drengage & 1250 & 2.90 & & 1.87 & & 2.90 & $\mathbf{M}$ & 1.34 & & 0 \\
\hline drill & 1300 & 6.07 & & 6.70 & . & 3.37 & & 7.00 & & 1 \\
\hline drug & 1240 & 6.171 & $\mathbf{M}$ & 6.53 & & 3.10 & $\mathbf{M}$ & 6.94 & & 1 \\
\hline dukely & 1826 & 2.43 & $\mathbf{F}$ & 3.94 & $\mathrm{~F}$ & 3.97 & . & 2.93 & $\mathbf{M}$ & 0 \\
\hline durzee & 1812 & 3.30 & . & 1.60 & & 3.40 & & 1.33 & & 0 \\
\hline Dutchify & 1680 & 3.20 & . & 2.37 & & 4.10 & $\mathrm{~F}$ & 2.36 & $\mathbf{M}$ & 0 \\
\hline earnestly & 1000 & 2.37 & . & 3.87 & $\mathrm{~F}$ & 5.00 & . & 6.57 & $\mathbf{M}$ & 1 \\
\hline echinuliform & 1846 & 3.23 & . & 1.20 & & 3.37 & & 1.50 & $\mathbf{M}$ & 0 \\
\hline educatable & 1868 & 2.87 & . & 4.20 & $\mathbf{F}$ & 5.44 & . & 5.57 & & 0 \\
\hline efficiently & 1628 & 2.57 & & 4.07 & & 6.07 & & 6.70 & & 1 \\
\hline eldern & 1200 & $3.60 \mathrm{I}$ & $\mathbf{M}$ & 3.10 & & 3.97 & . & 2.24 & $\mathbf{M}$ & 1 \\
\hline Elian & 1854 & 4.901 & $\mathbf{M}$ & 1.73 & M & 3.30 & . & 1.50 & & $\mathbf{0}$ \\
\hline eliminant & 1876 & 3.37 & . & 3.40 & 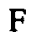 & 3.90 & . & 3.90 & F & $\mathbf{0}$ \\
\hline elocutionize & 1849 & 2.23 & & 2.17 & $\mathbf{M}$ & 2.80 & & 1.77 & & 0 \\
\hline embrocation & 1543 & 2.57 & $\mathrm{~F}$ & 1.50 & $\mathbf{M}$ & 3.44 & & 1.67 & & 0 \\
\hline emydian & 1854 & 3.00 & & 1.54 & $\mathbf{M}$ & 3.27 & & 1.30 & & 0 \\
\hline endangering & 1585 & 2.77 & $\mathbf{M}$ & 4.53 & . & 2.36 & $\mathbf{F}$ & 6.40 & & 1 \\
\hline engirdling & 1598 & 2.97 & . & 3.00 & & 3.37 & . & 2.67 & & 0 \\
\hline enregister & 1523 & 2.90 & & 2.40 & F & 3.87 & . & 2.93 & & 0 \\
\hline enroll & 1350 & 3.10 & $\mathbf{M}$ & 4.93 & $\mathrm{~F}$ & 4.83 & & 6.77 & & 1 \\
\hline entombing & 1564 & 3.37 & $\mathbf{F}$ & 4.03 & . & 2.63 & $\mathbf{M}$ & 4.10 & $\mathbf{M}$ & 0 \\
\hline epigone & 1866 & 3.40 & $\mathrm{~F}$ & 1.83 & . & 3.76 & . & 1.97 & & 0 \\
\hline equivalency & 1535 & 2.63 & & 4.30 & F & 4.77 & . & 5.74 & & 0 \\
\hline erythromycin & 1952 & 3.87 & . & 1.43 & & 3.43 & . & 2.07 & $\mathbf{M}$ & 0 \\
\hline escaping & 1325 & 3.80 & . & 6.57 & & 4.53 & . & 6.97 & . & 1 \\
\hline estoilee & 1730 & 2.87 & . & 1.67 & $\mathbf{M}$ & 3.20 & . & 1.57 & . & 0 \\
\hline evacuate & 1526 & 3.50 & . & 5.50 & $\mathrm{~F}$ & 2.53 & . & 6.67 & . & 1 \\
\hline evicted & 1604 & 3.83 & . & 5.37 & $\mathrm{~F}$ & 1.93 & . & 6.77 & . & 1 \\
\hline exarchate & 1561 & 2.87 & $\mathbf{F}$ & 1.47 & & 3.40 & . & 1.37 & . & 0 \\
\hline excretin & 1854 & 3.80 & . & 3.53 & $\mathbf{F}$ & 2.80 & . & 3.80 & . & 0 \\
\hline exhibition & 1432 & 5.40 & . & 5.97 & & 5.53 & . & 6.90 & . & 1 \\
\hline exhilarator & 1807 & 3.73 & . & 3.60 & F & 5.44 & & 4.87 & . & $\mathbf{0}$ \\
\hline experienceless & 1875 & 2.63 & . & 3.73 & F & 2.50 & . & 4.67 & & 0 \\
\hline exposure & 1605 & 3.93 & & 5.63 & . & 4.27 & . & 6.93 & & 1 \\
\hline expurgatory & 1625 & 2.60 & $\mathbf{M}$ & 2.10 & . & 3.23 & . & 3.03 & $\mathbf{M}$ & 0 \\
\hline extraneous & 1638 & 2.37 & & 3.70 & & 3.67 & & 5.20 & . & 1 \\
\hline face & 1290 & 6.63 & & 6.50 & $\mathrm{~F}$ & 5.70 & $\mathbf{F}$ & 7.00 & & 1 \\
\hline faceted & 1836 & 3.50 & $\mathrm{~F}$ & 3.40 & $\mathrm{~F}$ & 4.27 & $\mathrm{~F}$ & 4.60 & $\mathbf{M}$ & 0 \\
\hline fair & 888 & 3.93 & . & 5.63 & $\mathrm{~F}$ & 6.03 & . & 7.00 & . & 1 \\
\hline fallowing & 1450 & 3.37 & $\mathrm{~F}$ & 2.93 & $\mathbf{M}$ & 3.76 & . & 4.14 & & 0 \\
\hline fantasy & 1325 & 2.97 & $\mathbf{M}$ & 5.84 & 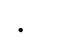 & 6.40 & & 6.90 & & 1 \\
\hline fascia & 1563 & 2.83 & . & 2.26 & & 3.80 & F & 2.70 & $\mathbf{M}$ & 0 \\
\hline fasten & 725 & 3.77 & . & 5.84 & $\mathrm{~F}$ & 4.77 & & 6.90 & & 1 \\
\hline favouritism & 1763 & 2.73 & . & 4.83 & . & 3.27 & $\mathrm{~F}$ & 6.40 & $\mathbf{M}$ & 0 \\
\hline feed & 950 & 5.33 & & 6.13 & & 5.33 & & 7.00 & & 1 \\
\hline feminity & 1386 & 3.40 & & 5.67 & F & 4.77 & $\mathbf{F}$ & 5.97 & $\mathbf{F}$ & 0 \\
\hline fescue & 1377 & 3.23 & $\mathbf{M}$ & 1.77 & . & 3.40 & $\mathbf{F}$ & 1.77 & . & $\mathbf{0}$ \\
\hline fetch & 1000 & 3.83 & $\mathbf{F}$ & 5.90 & . & 4.23 & . & 6.93 & . & 1 \\
\hline field & 1000 & 6.33 & & 6.63 & & 5.44 & & 6.97 & & 1 \\
\hline filler & 1496 & 4.40 & $\mathbf{M}$ & 5.47 & $\mathbf{F}$ & 3.30 & & 5.90 & $\mathbf{M}$ & 1 \\
\hline fingerful & 1889 & 3.70 & $\mathrm{~F}$ & 5.44 & $\mathbf{F}$ & 4.47 & $\mathbf{M}$ & 4.67 & $\mathbf{M}$ & 0 \\
\hline finish & 1350 & 3.50 & $\mathbf{M}$ & 5.90 & & 5.77 & $\mathbf{M}$ & 7.00 & & 1 \\
\hline finiteness & 1601 & 2.83 & . & 4.50 & $\mathbf{M}$ & 4.37 & $\mathbf{F}$ & 4.06 & $\mathbf{M}$ & 0 \\
\hline fishery & 1677 & 5.93 & & 6.23 & . & 4.34 & & 6.54 & $\mathbf{M}$ & 1 \\
\hline flair & 1340 & 3.10 & & 5.10 & & 5.47 & $\mathbf{F}$ & 6.50 & $\mathbf{M}$ & 1 \\
\hline flare-up & 1837 & 3.73 & $\mathbf{M}$ & 5.43 & & 3.47 & $\mathbf{F}$ & 5.90 & . & 0 \\
\hline flattery & 1320 & 3.30 & $F$ & 5.04 & $\mathbf{F}$ & 5.17 & $\mathbf{M}$ & 6.93 & & 1 \\
\hline flexional & 1833 & 2.70 & $\mathrm{~F}$ & 3.00 & & 4.27 & $\mathbf{F}$ & 2.57 & $\mathbf{M}$ & 0 \\
\hline floor & 888 & 6.80 & & 6.67 & $\mathbf{F}$ & 4.47 & $\mathbf{F}$ & 6.90 & & 1 \\
\hline flourocarbon & 1937 & 4.57 & $\mathbf{M}$ & 3.27 & $\mathbf{M}$ & 3.27 & & 3.83 & $\mathbf{M}$ & 1 \\
\hline flush & 1300 & 4.43 & . & 6.27 & . & 3.53 & . & 6.90 & . & 1 \\
\hline
\end{tabular}


APPENDIX (Continued)

\begin{tabular}{|c|c|c|c|c|c|c|c|c|c|c|}
\hline WORD & DOE & CONC & & IMAG & & GOOD & & FAM & & FREQ \\
\hline foil & 1300 & 5.77 & . & 6.14 & $\cdot$ & 3.30 & ${ }^{\circ}$ & 6.83 & . & 1 \\
\hline foot & 825 & 6.57 & . & 6.60 & $\mathbf{F}$ & 4.94 & . & 7.00 & & 1 \\
\hline fordable & 1611 & 2.87 & . & 2.57 & . & 4.53 & . & 3.60 & $\mathbf{M}$ & 0 \\
\hline foretoken & 888 & 2.90 & . & 2.57 & . & 3.70 & . & 3.83 & $\mathbf{F}$ & 0 \\
\hline forming & 1406 & 3.30 & . & 4.80 & . & 4.97 & . & 6.94 & . & 1 \\
\hline formulaic & 1882 & 3.17 & . & 2.93 & $\mathbf{M}$ & 4.23 & . & 2.60 & . & 1 \\
\hline forwardal & 1911 & 3.23 & . & 2.93 & $\mathrm{~F}$ & 4.23 & $\mathbf{F}$ & 2.47 & $\mathbf{F}$ & 0 \\
\hline foudroyant & 1840 & 2.87 & . & 1.50 & . & 3.13 & . & 1.40 & $\mathbf{M}$ & 0 \\
\hline framework & 1644 & 5.17 & . & 5.67 & . & 4.80 & . & 6.70 & . & 1 \\
\hline freight & 1463 & 5.60 & $\mathbf{M}$ & 6.20 & $\mathrm{~F}$ & 4.33 & $\mathbf{M}$ & 6.83 & . & 1 \\
\hline freshwater & 1528 & 6.07 & $\mathbf{M}$ & 6.47 & . & 6.20 & . & 6.80 & . & 1 \\
\hline frill & 1574 & 4.70 & . & 5.63 & $\mathrm{~F}$ & 4.53 & $\mathrm{~F}$ & 6.73 & $\cdot$ & 1 \\
\hline frowningly & 1556 & 2.87 & $\mathbf{F}$ & 5.34 & $\mathrm{~F}$ & 2.53 & $\mathbf{M}$ & 5.24 & $\mathbf{M}$ & 1 \\
\hline fulling & 1377 & 2.80 & . & 3.77 & . & 4.47 & $F$ & 3.87 & $\mathbf{F}$ & 0 \\
\hline furnished & 1473 & 4.93 & . & 6.30 & . & 5.64 & . & 7.00 & . & 1 \\
\hline futurology & 1946 & 2.73 & $F$ & 3.24 & & 4.40 & $\therefore$ & 2.50 & $\therefore$ & 0 \\
\hline gaff & 1300 & 4.10 & $\mathbf{M}$ & 3.60 & $\mathbf{M}$ & 3.13 & $\mathbf{M}$ & 3.37 & $\mathbf{M}$ & 1 \\
\hline gallize & 1888 & 2.47 & . & 1.80 & $\mathbf{M}$ & 3.34 & . & 1.57 & & 0 \\
\hline gape & 1220 & 4.17 & . & 5.13 & . & 3.27 & $\mathbf{M}$ & 5.37 & $\mathbf{M}$ & 1 \\
\hline gasping & 1440 & 4.60 & . & 6.13 & . & 2.20 & $\mathbf{M}$ & 6.87 & . & 1 \\
\hline gatch & 1886 & 2.90 & & 1.90 & . & 3.40 & . & 2.00 & $\mathbf{M}$ & 0 \\
\hline gearing & 1825 & 3.40 & $\mathbf{M}$ & 5.17 & $\mathbf{F}$ & 4.47 & . & 5.87 & . & 1 \\
\hline geniture & 1548 & 2.70 & $\mathbf{M}$ & 2.33 & . & 3.94 & . & 2.13 & . & 0 \\
\hline George & 1506 & 5.63 & $\mathbf{M}$ & 5.47 & $\mathbf{F}$ & 4.64 & . & 6.94 & . & 1 \\
\hline germiduct & 1885 & 3.13 & $\mathrm{~F}$ & 2.13 & . & 2.74 & $\mathbf{M}$ & 1.87 & . & 0 \\
\hline get & 1200 & 2.67 & . & 4.37 & . & 4.57 & . & 7.00 & . & 1 \\
\hline gibbsite & 1822 & 3.17 & $\mathbf{M}$ & 1.57 & . & 3.53 & . & 1.63 & . & 0 \\
\hline gird & 950 & 3.30 & $\mathbf{M}$ & 3.50 & $\mathbf{M}$ & 3.37 & . & 2.84 & . & 1 \\
\hline gladiatorism & 1860 & 2.70 & - & 3.53 & $\mathbf{M}$ & 3.34 & . & 3.00 & $\mathbf{M}$ & 0 \\
\hline glide & 1000 & 3.93 & $\mathbf{M}$ & 6.37 & . & 5.50 & $\mathbf{F}$ & 6.90 & . & 1 \\
\hline glum & 1460 & 3.53 & $\mathbf{M}$ & 5.44 & . & 2.24 & . & 5.87 & $\mathbf{M}$ & 1 \\
\hline glyceryl & 1845 & 4.70 & . & 3.43 & . & 3.74 & . & 4.77 & $\mathbf{F}$ & 0 \\
\hline go & 825 & 3.27 & . & 6.10 & . & 5.67 & . & 7.00 & . & 1 \\
\hline gold-colour & 1678 & 5.47 & . & 6.40 & $\mathbf{F}$ & 5.60 & . & 5.30 & . & 0 \\
\hline goon & 1921 & 5.03 & $\mathbf{M}$ & 5.77 & . & 2.77 & . & 5.80 & $\mathbf{M}$ & 1 \\
\hline goosified & 1837 & 2.70 & . & 2.87 & $\mathrm{~F}$ & 3.17 & . & 2.44 & . & $\mathbf{0}$ \\
\hline gowff & 1796 & 3.10 & $\mathbf{M}$ & 1.57 & . & 2.64 & . & 1.50 & . & 0 \\
\hline grallatorial & 1835 & 2.53 & . & 1.30 & . & 3.03 & . & 1.60 & . & 0 \\
\hline grass & 725 & 6.77 & . & 6.84 & . & 5.77 & . & 6.97 & . & 1 \\
\hline gratters & 1903 & 4.00 & . & 2.80 & . & 3.34 & . & 2.54 & . & 0 \\
\hline great & 888 & 2.67 & $\mathbf{M}$ & 4.73 & . & 6.44 & . & 7.00 & . & 1 \\
\hline greeting & 900 & 4.30 & . & 6.07 & $\mathrm{~F}$ & 6.07 & . & 7.00 & . & 1 \\
\hline griddle & 1225 & 5.23 & $\mathbf{F}$ & 5.40 & $\mathbf{F}$ & 4.17 & $\mathrm{~F}$ & 6.40 & . & 1 \\
\hline grinding & 1000 & 4.43 & $\mathrm{~F}$ & 6.03 & . & 2.93 & $\mathbf{M}$ & 6.60 & $\mathbf{M}$ & 1 \\
\hline grotesque & 1561 & 3.53 & . & 6.00 & . & 1.74 & . & 6.54 & . & 1 \\
\hline groupment & 1887 & 3.17 & $\mathbf{M}$ & 3.90 & . & 4.26 & F & 3.77 & . & 0 \\
\hline grudger & 1467 & 3.20 & . & 3.43 & $\mathrm{~F}$ & 2.53 & . & 3.27 & & 0 \\
\hline guib & 1774 & 2.87 & . & 1.77 & . & 2.87 & . & 2.13 & $\mathbf{M}$ & 0 \\
\hline gun-cotton & 1846 & 5.27 & . & 4.04 & . & 2.87 & . & 3.70 & $\mathbf{M}$ & 0 \\
\hline gyne & 1905 & 3.23 & $\mathbf{M}$ & 1.40 & . & 3.07 & . & 1.77 & $\mathbf{M}$ & 0 \\
\hline gyte & 1725 & 3.93 & $\mathbf{M}$ & 1.30 & . & 3.13 & . & 1.34 & . & 0 \\
\hline hagiology & 1807 & 3.23 & . & 1.57 & $\mathbf{M}$ & 3.50 & . & 1.40 & $\mathbf{M}$ & 0 \\
\hline hallo & 1781 & 3.60 & . & 4.50 & . & 5.23 & . & 3.84 & . & 1 \\
\hline hamartia & 1789 & 3.10 & . & 1.50 & $\mathbf{M}$ & 3.57 & . & 2.07 & $\mathbf{M}$ & 0 \\
\hline handily & 1611 & 2.90 & . & 4.03 & . & 5.20 & . & 5.94 & $\mathbf{M}$ & 0 \\
\hline hard & 971 & 4.93 & $\mathbf{M}$ & 6.37 & $\mathrm{~F}$ & 4.00 & . & 7.00 & . & 1 \\
\hline Harris & 1892 & 4.97 & $\mathbf{M}$ & 4.30 & $\mathbf{F}$ & 4.10 & $\mathbf{F}$ & 4.87 & $\mathbf{M}$ & 1 \\
\hline harsh & 1300 & 3.83 & $\mathbf{M}$ & 5.50 & . & 2.13 & . & 7.00 & . & 1 \\
\hline have & 800 & 2.23 & $\mathbf{M}$ & 3.43 & . & 4.93 & . & 7.00 & . & 1 \\
\hline headful & 1589 & 3.37 & . & 4.80 & $\mathbf{F}$ & 4.10 & . & 5.47 & & 0 \\
\hline headstock & 1731 & 5.03 & $\mathbf{M}$ & 3.97 & . & 3.70 & $\mathbf{M}$ & 3.80 & $\mathbf{F}$ & 1 \\
\hline
\end{tabular}


APPENDIX (Continued)

\begin{tabular}{|c|c|c|c|c|c|c|c|c|c|c|}
\hline WORD & DOE & CONC & & IMAG & & GOOD & & FAM & & FREQ \\
\hline heat & 700 & 5.93 & . & 6.20 & & 5.13 & . & 7.00 & & 1 \\
\hline heckelphone & 1905 & 4.20 & . & 2.90 & & 3.40 & . & 1.90 & & 0 \\
\hline hedgeling & 1787 & 4.03 & . & 2.87 & & 3.70 & . & 3.07 & & 0 \\
\hline help & 893 & 3.00 & . & 5.94 & F & 4.87 & . & 7.00 & & 1 \\
\hline herbary & 1548 & 3.57 & & 2.60 & $\mathbf{M}$ & 3.97 & 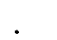 & 2.17 & & 0 \\
\hline Hessian & 1677 & 4.17 & $\mathbf{M}$ & 1.90 & $\mathbf{M}$ & 3.33 & . & 2.17 & $\mathbf{M}$ & 1 \\
\hline heterochronous & 1854 & 2.83 & . & 1.73 & $\mathbf{M}$ & 3.47 & & 1.77 & $\mathbf{M}$ & 0 \\
\hline hierocracy & 1749 & 2.63 & . & 3.17 & . & 3.33 & M & 3.50 & $\mathrm{~F}$ & 0 \\
\hline hinged & 1672 & 4.17 & . & 5.37 & & 4.07 & . & 6.60 & & 1 \\
\hline hippeastrum & 1821 & 3.23 & . & 1.37 & $\mathbf{M}$ & 3.47 & . & 1.37 & $\mathbf{M}$ & 0 \\
\hline Hobbesian & 1776 & 4.00 & . & 1.93 & $\mathbf{F}$ & 3.60 & & 2.30 & $\mathbf{M}$ & 0 \\
\hline holding & 1225 & 4.47 & . & 5.80 & $\mathbf{F}$ & 5.00 & $\mathrm{~F}$ & 6.94 & & 1 \\
\hline holster & 1663 & 6.07 & . & 5.63 & & 3.67 & $\mathrm{~F}$ & 6.44 & $\mathbf{M}$ & 1 \\
\hline homoeomerian & 1875 & 3.57 & & 1.53 & $\mathbf{M}$ & 3.30 & & 1.40 & . & 0 \\
\hline hoodooism & 1881 & 2.67 & $\mathbf{M}$ & 2.77 & $\mathbf{M}$ & 2.57 & & 2.26 & . & 0 \\
\hline hooked & 1000 & 4.33 & & 6.20 & . & 3.37 & & 6.90 & . & 1 \\
\hline hop & 1000 & 4.93 & & 6.47 & F & 4.94 & $\mathrm{~F}$ & 7.00 & & 1 \\
\hline horse-fish & 1723 & 5.73 & & 5.17 & F & 3.77 & . & 3.77 & F & 0 \\
\hline hot & 825 & 5.40 & $\mathbf{M}$ & 6.47 & . & 4.97 & . & 7.00 & . & 1 \\
\hline house & 950 & 6.77 & & 6.84 & . & 5.84 & & 6.97 & . & 1 \\
\hline hulk & 1000 & 4.43 & $\mathbf{M}$ & 6.07 & . & 4.20 & $\mathbf{M}$ & 6.90 & . & 1 \\
\hline hundi & 1619 & 3.20 & $\mathbf{M}$ & 3.17 & . & 3.40 & & 2.07 & & 0 \\
\hline hunting & 950 & 4.43 & $\mathrm{~F}$ & 6.50 & & 3.34 & $\mathbf{M}$ & 7.00 & & 1 \\
\hline hydraulicity & 1843 & 3.27 & & 3.03 & $\mathbf{M}$ & 4.37 & . & 2.97 & $\mathbf{M}$ & 0 \\
\hline hyperchromatic & 1894 & 3.13 & $\mathrm{~F}$ & 2.20 & $\mathbf{M}$ & 3.23 & . & 2.77 & $\mathbf{M}$ & 0 \\
\hline hyphomycetous & 1887 & 2.93 & $\mathrm{~F}$ & 1.20 & & 3.14 & . & 1.26 & . & 0 \\
\hline ice & 900 & 6.63 & & 6.74 & & 4.60 & . & 7.00 & & 1 \\
\hline icespar & 1816 & 4.03 & $\mathbf{M}$ & 2.77 & $\mathbf{F}$ & 3.73 & . & 2.50 & & 0 \\
\hline igasuric & 1830 & 2.43 & . & 1.40 & $\mathbf{M}$ & 2.90 & & 1.37 & & 0 \\
\hline ill-willy & 1500 & 2.33 & & 2.67 & $\mathbf{M}$ & 2.97 & $\mathbf{F}$ & 2.50 & & 0 \\
\hline imaged & 1718 & 3.07 & $\mathbf{M}$ & 4.07 & $\mathbf{M}$ & 5.34 & . & 6.00 & & 0 \\
\hline impaint & 1596 & 3.10 & . & 3.13 & . & 3.77 & & 2.50 & $\mathrm{~F}$ & 0 \\
\hline impeller & 1685 & 3.87 & & 2.90 & . & 3.47 & $\mathbf{M}$ & 3.30 & $\mathbf{M}$ & 0 \\
\hline implant & 1541 & 4.60 & $\mathbf{M}$ & 5.34 & . & 4.27 & 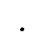 & 6.27 & $\mathbf{M}$ & 1 \\
\hline improbably & 1646 & 2.23 & . & 2.90 & & 3.17 & . & 5.06 & . & 1 \\
\hline inagglutinable & 1919 & 2.33 & . & 1.80 & $\mathbf{M}$ & 3.30 & . & 1.87 & $\mathbf{M}$ & 0 \\
\hline inappeasable & 1840 & 2.00 & & 2.74 & $\mathrm{~F}$ & 2.67 & . & 4.10 & & 0 \\
\hline inclinable & 1449 & 2.77 & $\mathbf{M}$ & 3.50 & . & 3.77 & & 3.60 & $\mathbf{M}$ & 0 \\
\hline inconvincible & 1646 & 2.73 & & 3.60 & & 3.03 & $\mathbf{M}$ & 5.53 & & 0 \\
\hline indenture & 1300 & 4.00 & $\mathbf{M}$ & 4.03 & $\mathbf{F}$ & 3.07 & $\mathbf{M}$ & 4.04 & & 1 \\
\hline indispose & 1657 & 2.47 & . & 3.03 & & 3.20 & . & 4.94 & & 0 \\
\hline indumentum & 1847 & 2.77 & . & 1.50 & $\mathbf{M}$ & 3.57 & . & 1.67 & & 0 \\
\hline ineliminable & 1875 & 2.50 & & 2.33 & . & 3.23 & . & 3.04 & & $\mathbf{0}$ \\
\hline infilled & 1849 & 3.20 & $\mathbf{M}$ & 3.37 & . & 3.64 & . & 3.37 & & $\mathbf{0}$ \\
\hline ingenerate & 1528 & 2.83 & . & 2.34 & & 3.43 & . & 3.50 & $\mathrm{~F}$ & 0 \\
\hline ink-berry & 1850 & 5.27 & . & 4.17 & $\mathbf{F}$ & 4.30 & . & 2.50 & $\mathbf{M}$ & 0 \\
\hline inorganic & 1794 & 3.93 & $\mathbf{M}$ & 4.40 & $\mathbf{M}$ & 3.90 & . & 5.74 & . & 1 \\
\hline inquisitively & 1631 & 2.53 & $\mathrm{~F}$ & 3.97 & $\mathrm{~F}$ & 5.00 & . & 5.74 & . & 1 \\
\hline inspiration & 1303 & 2.33 & $\mathbf{M}$ & 4.67 & $\mathbf{M}$ & 6.20 & . & 6.83 & . & 1 \\
\hline integer & 1509 & 4.67 & . & 4.60 & $\mathrm{~F}$ & 3.80 & . & 4.93 & & 1 \\
\hline interchangeability & 1805 & 2.37 & & 4.06 & . & 5.33 & & 5.77 & . & 1 \\
\hline Interglossa & 1943 & 4.17 & $\mathbf{M}$ & 1.70 & & 3.47 & $\mathbf{F}$ & 1.50 & & 0 \\
\hline intermittent & 1603 & 2.67 & $\mathrm{~F}$ & 4.37 & $\mathbf{M}$ & 3.87 & . & 5.84 & $\mathbf{M}$ & 1 \\
\hline intinction & 1559 & 2.77 & $\mathbf{M}$ & 2.13 & . & 3.90 & . & 2.80 & . & 0 \\
\hline invariance & 1878 & 2.30 & & 2.87 & . & 3.24 & . & 4.34 & & 0 \\
\hline inversion & 1551 & 3.53 & $\mathbf{M}$ & 4.67 & 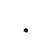 & 3.60 & . & 6.47 & $\mathbf{M}$ & 1 \\
\hline ion & 1834 & 5.03 & $\mathbf{M}$ & 4.67 & F & 4.40 & . & 6.07 & & 1 \\
\hline irrepleviable & 1543 & 2.53 & & 1.93 & . & 3.17 & $\mathbf{M}$ & 1.84 & & 0 \\
\hline isocentre & 1931 & 3.27 & $\mathbf{F}$ & 2.47 & & 4.07 & & 2.77 & $\mathbf{M}$ & 0 \\
\hline issue & 1308 & 3.23 & $\mathbf{M}$ & 4.97 & $\mathbf{F}$ & 4.57 & $\mathbf{M}$ & 7.00 & . & 1 \\
\hline jack & 1362 & 6.20 & & 5.97 & $\mathrm{~F}$ & 4.20 & $\mathrm{~F}$ & 6.93 & & 1 \\
\hline
\end{tabular}


APPENDIX (Continued)

\begin{tabular}{|c|c|c|c|c|c|c|c|c|c|c|}
\hline WORD & DOE & CONC & & IMAG & & GOOD & & FAM & & FREQ \\
\hline jack-boot & 1686 & 4.80 & & 3.50 & & 3.43 & & 3.37 & $\mathbf{M}$ & 0 \\
\hline Jagatai & 1843 & 3.97 & $\mathbf{M}$ & 1.44 & & 3.53 & . & 1.30 & & 1 \\
\hline jar-fly & 1880 & 5.17 & & 4.53 & $\mathbf{M}$ & 2.97 & . & 3.24 & $\mathbf{F}$ & 0 \\
\hline jetty & 1412 & 3.77 & $\mathbf{M}$ & 3.87 & . & 4.23 & & 4.04 & $\mathbf{M}$ & 1 \\
\hline Jew & 1175 & 5.73 & . & 6.13 & . & 4.03 & F & 6.84 & . & 1 \\
\hline joint & 1290 & 5.50 & & 6.63 & . & 4.23 & $\mathbf{M}$ & 7.00 & . & 1 \\
\hline Joule-Kelvin & 1909 & 4.20 & & 2.50 & & 3.40 & . & 1.94 & . & 0 \\
\hline judgement & 1225 & 2.87 & $\mathbf{M}$ & 4.80 & $\mathbf{M}$ & 4.64 & . & 6.94 & . & 1 \\
\hline just & 1250 & 2.67 & & 3.67 & $\mathbf{M}$ & 5.37 & . & 6.94 & . & 1 \\
\hline kallikrein & 1930 & 2.93 & $\mathbf{M}$ & 1.34 & $\mathbf{M}$ & 3.37 & . & 1.37 & & 0 \\
\hline keen & 897 & 2.40 & & 4.63 & & 5.70 & . & 6.57 & $\mathbf{M}$ & 1 \\
\hline Kenite & 1000 & 4.00 & $\mathbf{M}$ & 1.47 & $\mathbf{M}$ & 3.50 & . & 1.26 & & 0 \\
\hline kevel & 1251 & 3.50 & . & 1.73 & . & 3.47 & . & 1.57 & $\mathbf{M}$ & 0 \\
\hline kiley & 1945 & 3.20 & & 1.57 & . & 3.27 & & 1.80 & $\mathbf{M}$ & 0 \\
\hline kindred & 1175 & 3.50 & $\mathbf{M}$ & 4.10 & . & 4.84 & F & 5.17 & & 1 \\
\hline klep & 1889 & 3.47 & $\mathrm{~F}$ & 2.30 & $\mathbf{M}$ & 3.23 & . & 2.07 & $\mathbf{M}$ & 0 \\
\hline klooch & 1837 & 2.83 & & 1.77 & $\mathbf{M}$ & 2.97 & . & 1.50 & . & 0 \\
\hline knacker & 1380 & 3.77 & $\mathrm{~F}$ & 2.54 & & 4.03 & . & 3.17 & & 0 \\
\hline knottiness & 1607 & 3.50 & . & 4.77 & $\mathbf{F}$ & 3.24 & . & 4.23 & & 0 \\
\hline komfoor & 1841 & 3.03 & . & 1.50 & $\mathbf{M}$ & 3.47 & & 1.34 & $\mathbf{M}$ & 0 \\
\hline labially & 1798 & 2.53 & . & 1.87 & . & 3.63 & $\mathbf{M}$ & 2.37 & $\mathbf{M}$ & 0 \\
\hline labourer & 1325 & 6.00 & . & 6.53 & . & 4.07 & F & 6.80 & . & 1 \\
\hline lagging & 1593 & 3.40 & $\mathbf{M}$ & 5.03 & & 2.77 & . & 6.20 & & 0 \\
\hline land-line & 1865 & 4.43 & $\mathrm{~F}$ & 4.00 & $\mathrm{~F}$ & 4.07 & . & 3.57 & $\mathbf{F}$ & 0 \\
\hline landaulet & 1794 & 3.47 & . & 1.63 & $\mathbf{M}$ & 3.84 & . & 1.57 & & 0 \\
\hline lapsing & 1663 & 3.40 & . & 3.87 & . & 2.97 & . & 5.23 & $\mathbf{M}$ & 1 \\
\hline latency & 1638 & 2.77 & . & 2.80 & $\mathbf{M}$ & 3.54 & . & 6.17 & . & 1 \\
\hline laughing & 1300 & 4.97 & . & 6.80 & . & 6.70 & & 6.97 & & 1 \\
\hline lavic & 1835 & 2.80 & . & 1.90 & . & 3.10 & & 1.77 & $\mathbf{M}$ & 0 \\
\hline layer & 1382 & 4.90 & . & 6.07 & . & 4.50 & $\mathbf{F}$ & 6.97 & . & 1 \\
\hline leap & 897 & 4.60 & $\mathbf{F}$ & 6.43 & . & 4.83 & . & 7.00 & . & 1 \\
\hline lechatelierite & 1916 & 3.40 & $\mathbf{M}$ & 1.17 & . & 3.20 & & 1.23 & . & 0 \\
\hline leech & 900 & 6.50 & . & 6.20 & & 1.87 & & 6.33 & . & 1 \\
\hline lending & 1340 & 3.57 & . & 5.60 & & 4.23 & . & 6.84 & & 1 \\
\hline lethargy & 1374 & 2.90 & & 3.67 & $\mathbf{M}$ & 2.70 & . & 5.06 & $\mathbf{M}$ & 1 \\
\hline Leucadian & 1615 & 3.97 & $\mathbf{M}$ & 1.53 & $\mathbf{M}$ & 3.10 & & 1.40 & & 0 \\
\hline liberalistic & 1836 & 2.50 & . & 3.74 & $\mathrm{~F}$ & 4.40 & $\mathbf{M}$ & 5.24 & . & 0 \\
\hline lieutenant-general & 1489 & 5.97 & & 6.00 & & 4.23 & . & 6.00 & . & $\mathbf{0}$ \\
\hline light & 825 & 6.37 & $\mathbf{F}$ & 6.50 & & 6.03 & $\mathbf{F}$ & 7.00 & & 1 \\
\hline light-skirts & 1597 & 5.10 & & 5.33 & & 4.53 & . & 4.00 & $\mathbf{M}$ & 0 \\
\hline limiter & 1377 & 3.87 & $\mathbf{F}$ & 3.44 & $\mathbf{F}$ & 2.87 & & 4.90 & $\mathbf{F}$ & 0 \\
\hline linger & 1300 & 3.20 & & 5.54 & $\mathbf{F}$ & 3.63 & $\mathbf{F}$ & 6.74 & . & 1 \\
\hline link & 931 & 4.93 & $\mathbf{M}$ & 5.87 & . & 4.87 & . & 6.90 & & 1 \\
\hline lintel & 1388 & 3.63 & & 2.20 & & 4.23 & . & 2.50 & $\mathbf{M}$ & 1 \\
\hline lithe & 888 & 3.77 & & 3.07 & $\mathbf{M}$ & 3.54 & . & 3.87 & $\mathbf{M}$ & 1 \\
\hline livid & 1622 & 3.53 & & 3.54 & $\mathrm{~F}$ & 3.44 & . & 4.94 & $\mathbf{M}$ & 1 \\
\hline lobbyist & 1863 & 5.23 & $\mathbf{M}$ & 5.23 & & 3.47 & . & 5.77 & $\mathbf{M}$ & 0 \\
\hline lock & 700 & 6.13 & & 6.33 & $\mathbf{F}$ & 3.67 & . & 6.97 & . & 1 \\
\hline loiterer & 1530 & 5.70 & $F$ & 6.20 & $\mathbf{F}$ & 2.57 & . & 5.70 & & 0 \\
\hline lonnin & 1808 & 3.03 & . & 1.27 & . & 3.00 & . & 1.40 & . & 0 \\
\hline look & 897 & 4.10 & $\cdot$ & 5.60 & . & 5.50 & . & 7.00 & . & 1 \\
\hline loricate & 1623 & 2.67 & & 1.47 & . & 3.57 & . & 1.60 & . & 0 \\
\hline lover & 1225 & 5.67 & F & 6.67 & & 6.50 & . & 7.00 & & 1 \\
\hline ludicrously & 1678 & 2.57 & . & 3.73 & & 2.90 & . & 5.14 & $\mathbf{F}$ & 1 \\
\hline Luiseno & 1858 & 4.53 & & 1.37 & $\mathbf{M}$ & 3.13 & . & 1.27 & . & 0 \\
\hline Lusian & 1776 & 4.33 & $\mathbf{M}$ & 1.77 & . & 3.30 & . & 1.50 & & 0 \\
\hline mace & 1297 & 5.93 & & 5.44 & & 2.67 & $\mathrm{~F}$ & 5.50 & $\mathbf{M}$ & 1 \\
\hline machinability & 1921 & 3.13 & & 3.87 & $\mathrm{~F}$ & 4.00 & $\mathbf{M}$ & 4.23 & & 1 \\
\hline magical & 1555 & 3.03 & $\mathbf{M}$ & 6.07 & $\mathbf{F}$ & 5.77 & . & 6.84 & $\cdot$ & 1 \\
\hline maimai & 1863 & 3.63 & $\mathbf{M}$ & 2.37 & & 3.50 & & 1.53 & & 0 \\
\hline maiming & 1400 & 3.63 & $\mathbf{F}$ & 4.80 & $\mathrm{~F}$ & 1.94 & & 4.97 & $\mathbf{M}$ & 1 \\
\hline
\end{tabular}


APPENDIX (Continued)

\begin{tabular}{|c|c|c|c|c|c|c|c|c|c|c|}
\hline WORD & DOE & CONC & & IMAG & & GOOD & & FAM & & FREQ \\
\hline make & 888 & 2.43 & & 4.57 & F & 5.03 & . & 7.00 & & 1 \\
\hline malleableness & 1644 & 3.53 & $\mathbf{M}$ & 3.00 & & 3.83 & & 3.90 & $\mathbf{M}$ & 0 \\
\hline man-child & 1400 & 5.00 & & 4.97 & $\mathbf{F}$ & 4.47 & . & 4.97 & $\mathbf{M}$ & 0 \\
\hline manifest & 1374 & 3.00 & & 3.83 & $\mathbf{M}$ & 4.40 & . & 6.20 & $\mathbf{M}$ & 1 \\
\hline manner & 1175 & 2.97 & & 4.83 & $\mathrm{~F}$ & 5.17 & . & 6.97 & . & 1 \\
\hline marasmolite & 1851 & 3.33 & & 1.57 & $\mathbf{M}$ & 3.53 & . & 1.44 & & 0 \\
\hline markedness & 1846 & 2.77 & & 3.33 & . & 3.60 & & 4.84 & $\mathbf{M}$ & 0 \\
\hline marl & 1372 & 3.47 & $\mathbf{F}$ & 1.80 & & 3.00 & $\mathbf{M}$ & 1.94 & & 1 \\
\hline Martinist & 1589 & 4.23 & & 2.17 & & 3.37 & & 1.80 & $\mathbf{M}$ & 0 \\
\hline master & 888 & 4.83 & $\mathbf{M}$ & 5.73 & . & 3.93 & $\mathrm{~F}$ & 7.00 & . & 1 \\
\hline masting & 1627 & 2.73 & . & 3.20 & $\mathbf{F}$ & 3.97 & $\mathbf{M}$ & 4.03 & & 0 \\
\hline math & 963 & 4.60 & & 6.47 & & 3.73 & $\mathrm{~F}$ & 7.00 & & 1 \\
\hline matin & 1250 & 2.90 & $\mathbf{M}$ & 2.90 & $\mathbf{F}$ & 3.57 & $\mathbf{M}$ & 2.50 & & 0 \\
\hline may & 700 & 2.17 & . & 4.37 & & 4.70 & $\mathrm{~F}$ & 6.97 & & 1 \\
\hline measurability & 1697 & 2.87 & . & 4.40 & $\mathbf{F}$ & 4.90 & . & 5.97 & & $\mathbf{0}$ \\
\hline Mediterranean & 1594 & 5.37 & & 6.33 & $\mathbf{F}$ & 5.57 & . & 6.90 & & 1 \\
\hline megaphone & 1878 & 6.00 & $\mathbf{M}$ & 5.40 & $\mathrm{~F}$ & 4.20 & . & 6.07 & $\mathbf{M}$ & 1 \\
\hline mellifluent & 1601 & 2.73 & & 1.67 & & 4.00 & . & 1.67 & & $\mathbf{0}$ \\
\hline menstruate & 1382 & 5.17 & $\mathrm{~F}$ & 6.13 & $\mathbf{F}$ & 3.07 & . & 6.77 & & 0 \\
\hline meroblast & 1870 & 3.07 & . & 2.10 & $\mathbf{M}$ & 3.47 & . & 1.53 & $\mathbf{M}$ & $\mathbf{0}$ \\
\hline mesocratic & 1857 & 3.13 & . & 1.40 & . & 3.10 & . & 1.90 & $\mathbf{M}$ & $\mathbf{0}$ \\
\hline metallographic & 1838 & 3.23 & . & 2.17 & . & 3.83 & . & 1.97 & $\mathrm{~F}$ & 0 \\
\hline mew & 725 & 3.67 & & 5.00 & . & 4.77 & . & 5.63 & $\mathrm{~F}$ & 1 \\
\hline Meyerbeerian & 1890 & 4.17 & $\mathbf{M}$ & 1.37 & & 3.27 & . & 1.20 & . & $\mathbf{0}$ \\
\hline mid-line & 1868 & 4.50 & . & 5.00 & $F$ & 3.90 & . & 5.17 & & 0 \\
\hline midgy & 1806 & 2.80 & $\mathbf{M}$ & 2.30 & & 3.40 & & 2.00 & & $\mathbf{0}$ \\
\hline mill & 931 & 6.53 & . & 6.30 & $\mathrm{~F}$ & 4.37 & $\mathbf{M}$ & 6.90 & . & 1 \\
\hline mineral & 1390 & 6.17 & . & 6.43 & & 4.90 & & 6.97 & . & 1 \\
\hline minimally & 1935 & 2.30 & . & 3.90 & $\mathrm{~F}$ & 3.10 & $\mathbf{F}$ & 5.97 & & 1 \\
\hline mird & 1614 & 3.37 & $\mathbf{M}$ & 1.63 & $\mathbf{M}$ & 3.20 & $\mathbf{M}$ & 1.67 & $\mathbf{M}$ & 0 \\
\hline misdrive & 1330 & 2.73 & . & 4.17 & $\mathbf{F}$ & 2.80 & . & 3.67 & $\mathbf{F}$ & 0 \\
\hline missed & 1599 & 3.40 & $\mathbf{M}$ & 5.44 & & 2.70 & . & 6.94 & . & 1 \\
\hline mitriform & 1830 & 3.07 & . & 1.67 & $\mathbf{M}$ & 3.47 & . & 1.47 & & 0 \\
\hline mnemotechnist & 1891 & 4.17 & . & 1.83 & $\mathbf{M}$ & 3.17 & & 1.60 & $\mathbf{M}$ & 0 \\
\hline moderating & 1673 & 2.50 & . & 3.74 & . & 5.10 & $\mathbf{M}$ & 6.40 & . & 1 \\
\hline mollifying & 1532 & 2.80 & . & 2.47 & . & 3.47 & . & 3.04 & . & 0 \\
\hline monchiquite & 1891 & 3.10 & & 1.43 & & 3.33 & . & 1.37 & & 0 \\
\hline monkish & 1546 & 2.87 & & 4.37 & $\mathrm{~F}$ & 4.10 & . & 4.67 & $\mathbf{M}$ & 1 \\
\hline Montenegrin & 1840 & 4.43 & $\mathbf{M}$ & 1.59 & . & 3.47 & . & 1.40 & $\mathbf{M}$ & 1 \\
\hline monumentalism & 1902 & 2.50 & & 3.37 & & 4.57 & & 4.14 & . & 0 \\
\hline moralize & 1450 & 2.20 & $\mathbf{M}$ & 3.50 & $\mathbf{F}$ & 4.63 & $\mathbf{M}$ & 5.44 & & 0 \\
\hline mortar & 1000 & 6.17 & $\mathbf{M}$ & 5.07 & & 3.33 & $\mathbf{M}$ & 5.70 & $\mathbf{M}$ & 1 \\
\hline mothed & 1835 & 3.43 & . & 3.20 & $\mathbf{F}$ & 2.93 & & 3.03 & . & 0 \\
\hline motion & 1374 & 4.37 & & 5.60 & $\mathbf{M}$ & 5.40 & $\mathrm{~F}$ & 7.00 & . & 1 \\
\hline motto & 1589 & 4.00 & $\mathbf{M}$ & 5.10 & . & 4.76 & . & 6.60 & & 1 \\
\hline mouse & 700 & 6.80 & . & 6.77 & . & 4.07 & . & 7.00 & & 1 \\
\hline muckraking & 1879 & 3.50 & . & 3.28 & . & 2.87 & & 3.64 & $\mathbf{M}$ & $\mathbf{0}$ \\
\hline mucus & 1661 & 6.17 & . & 6.30 & . & 1.97 & & 6.47 & . & 1 \\
\hline multurer & 1580 & 3.80 & & 1.90 & . & 3.44 & . & 1.80 & & 0 \\
\hline mummy & 1400 & 6.40 & & 6.60 & & 4.97 & & 6.30 & $\mathbf{M}$ & 1 \\
\hline muser & 1382 & 3.57 & $\mathbf{M}$ & 2.97 & . & 3.47 & & 3.26 & $\mathbf{M}$ & 0 \\
\hline mutterer & 1552 & 4.22 & $\mathrm{~F}$ & 5.06 & . & 2.64 & . & 5.10 & $\mathbf{M}$ & 0 \\
\hline myoid & 1857 & 3.23 & & 1.40 & $\mathbf{M}$ & 3.24 & $\mathbf{M}$ & 1.43 & $\mathbf{M}$ & 0 \\
\hline nacket & 1500 & 3.33 & $\mathbf{M}$ & 2.13 & . & 3.40 & . & 2.10 & $\mathbf{M}$ & 0 \\
\hline narratress & 1798 & 4.07 & & 3.67 & $F$ & 4.27 & & 3.60 & $F$ & 0 \\
\hline nartjie & 1790 & 2.87 & & 1.20 & & 3.13 & & 1.37 & $\mathbf{M}$ & 0 \\
\hline nauseation & 1628 & 3.77 & & 4.63 & $\mathrm{~F}$ & 1.83 & $\mathbf{M}$ & 5.04 & $F$ & 0 \\
\hline necrolatry & 1826 & 3.13 & $\mathbf{M}$ & 1.60 & $\mathbf{M}$ & 3.07 & $\mathbf{M}$ & 1.47 & $\mathbf{M}$ & 0 \\
\hline necropolitan & 1892 & 3.60 & $\mathbf{M}$ & 1.77 & $\mathbf{M}$ & 3.07 & & 1.70 & & 0 \\
\hline nemertean & 1861 & 3.07 & & 1.47 & $\mathbf{M}$ & 3.17 & $\mathbf{M}$ & 1.54 & $\mathbf{M}$ & 0 \\
\hline Netherlandic & 1902 & 4.20 & F & 4.13 & $\mathrm{~F}$ & 4.24 & & 3.27 & $\mathbf{M}$ & 0 \\
\hline
\end{tabular}


APPENDIX (Continued)

\begin{tabular}{|c|c|c|c|c|c|c|c|c|c|c|}
\hline WORD & DOE & CONC & & IMAG & & GOOD & & FAM & & FREQ \\
\hline neurosal & 1884 & 2.60 & $\mathrm{~F}$ & 2.27 & $\mathbf{M}$ & 3.57 & $\mathbf{M}$ & 2.37 & $\mathbf{M}$ & 0 \\
\hline niacinamide & 1951 & 4.33 & $\mathbf{M}$ & 1.97 & $\mathbf{M}$ & 3.00 & $\mathrm{~F}$ & 3.80 & $\mathbf{M}$ & $\mathbf{0}$ \\
\hline nick & 1225 & 5.30 & . & 5.43 & & 3.37 & . & 6.37 & & 1 \\
\hline nihilistic & 1857 & 2.60 & $\mathbf{M}$ & 2.03 & $\mathbf{M}$ & 3.13 & & 3.30 & $\mathbf{M}$ & 1 \\
\hline Nkrumahism & 1960 & 3.93 & $\mathbf{M}$ & 1.37 & $\mathbf{M}$ & 2.93 & 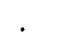 & 1.17 & & 0 \\
\hline noblewoman & 1575 & 5.53 & $\mathbf{M}$ & 5.37 & & 5.03 & . & 5.10 & $\mathbf{F}$ & 1 \\
\hline non-condensing & 1841 & 3.27 & . & 3.94 & & 4.07 & . & 4.76 & . & 0 \\
\hline non-object & 1914 & 2.50 & . & 3.03 & & 3.70 & . & 4.52 & . & 0 \\
\hline north-polar & 1748 & 3.97 & & 5.50 & $\mathrm{~F}$ & 4.50 & . & 4.43 & F & 0 \\
\hline nothomorph & 1939 & 3.47 & $\mathbf{M}$ & 1.50 & $\mathbf{M}$ & 2.90 & 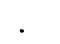 & 1.40 & & 0 \\
\hline notoriously & 1512 & 2.60 & . & 3.54 & & 3.60 & . & 6.20 & . & 1 \\
\hline nulliverse & 1847 & 2.47 & . & 2.47 & & 2.80 & . & 1.54 & . & 0 \\
\hline nutritionalist & 1956 & 5.23 & & 5.08 & $\mathrm{~F}$ & 5.43 & & 5.60 & $\mathbf{F}$ & 0 \\
\hline nymphal & 1622 & 2.97 & $\mathbf{M}$ & 3.04 & & 3.87 & $\mathbf{M}$ & 3.14 & & 0 \\
\hline obligationary & 1880 & 2.47 & . & 2.97 & 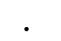 & 3.73 & $\mathbf{M}$ & 4.00 & $\mathbf{F}$ & 0 \\
\hline obviate & 1598 & 2.73 & $\mathrm{~F}$ & 2.27 & . & 3.43 & . & 3.04 & $\mathbf{M}$ & 1 \\
\hline ocean & 1290 & 6.63 & & 6.84 & & 6.20 & & 6.97 & . & 1 \\
\hline octupole & 1929 & 3.63 & F & 2.20 & $\mathbf{M}$ & 4.07 & & 1.77 & . & 0 \\
\hline odmyl & 1866 & 3.10 & & 1.27 & & 3.20 & & 1.23 & . & 0 \\
\hline officially & 1790 & 2.53 & . & 4.23 & $\mathbf{M}$ & 4.50 & $\mathbf{M}$ & 6.83 & . & 1 \\
\hline oiling & 1440 & 3.80 & & 6.03 & & 4.43 & & 6.54 & . & 1 \\
\hline oligoclase & 1832 & 2.73 & M & 1.37 & $\mathbf{M}$ & 3.33 & $\mathbf{M}$ & 1.40 & . & 0 \\
\hline on & 876 & 2.70 & & 5.63 & & 5.10 & & 7.00 & & 1 \\
\hline one-sided & 1813 & 3.83 & . & 5.23 & & 2.64 & & 6.37 & & 1 \\
\hline open & 825 & 4.20 & & 6.44 & & 5.57 & . & 6.97 & & 1 \\
\hline operetta & 1770 & 5.13 & F & 4.97 & $\mathrm{~F}$ & 4.93 & & 4.70 & & 1 \\
\hline ora & 920 & 3.20 & & 3.47 & & 4.63 & $\mathbf{M}$ & 3.40 & F & 0 \\
\hline orchiectomy & 1894 & 3.10 & . & 1.44 & $\mathbf{M}$ & 3.04 & $\mathbf{M}$ & 1.47 & & 0 \\
\hline ordinate & 1374 & 2.93 & . & 3.20 & . & 3.70 & $\mathbf{F}$ & 4.90 & & 1 \\
\hline oronoco & 1706 & 3.23 & & 1.30 & & 3.47 & . & 1.90 & $\mathbf{M}$ & 0 \\
\hline Ortygian & 1640 & 4.23 & $\mathbf{M}$ & 1.53 & $\mathbf{M}$ & 3.33 & & 1.14 & & 0 \\
\hline other & 735 & 2.77 & . & 3.50 & & 4.37 & F & 6.97 & & 1 \\
\hline outed & 1500 & 2.63 & . & 3.43 & $\mathbf{M}$ & 3.17 & F & 4.00 & & 0 \\
\hline outland & 950 & 4.47 & & 4.76 & & 4.30 & $\mathbf{F}$ & 5.43 & & 0 \\
\hline outsider & 1800 & 5.07 & $\mathrm{~F}$ & 5.67 & $\mathrm{~F}$ & 3.00 & $\mathbf{M}$ & 6.80 & & 1 \\
\hline over-officious & 1610 & 2.33 & & 2.80 & . & 2.60 & & 2.67 & $\mathbf{M}$ & 0 \\
\hline overburn & 1616 & 3.90 & $\mathbf{M}$ & 5.37 & . & 2.77 & $\mathbf{M}$ & 4.87 & & 0 \\
\hline overfulfil & 1398 & 2.80 & $\mathbf{M}$ & 4.23 & . & 4.83 & & 4.84 & & 0 \\
\hline overween & 1220 & 2.97 & & 2.67 & . & 2.87 & & 2.94 & $F$ & 0 \\
\hline Oxford & 1590 & 5.67 & $\mathbf{M}$ & 5.77 & . & 5.04 & $\mathbf{M}$ & 6.40 & & 1 \\
\hline ozonizer & 1875 & 4.03 & $\mathbf{M}$ & 2.74 & . & 3.40 & $\mathbf{F}$ & 2.30 & M & 0 \\
\hline packsaddle & 1388 & 5.57 & $\mathbf{M}$ & 4.77 & . & 4.33 & $\mathbf{F}$ & 3.74 & F & 0 \\
\hline paddle & 1407 & 6.57 & & 6.84 & - & 4.77 & $\mathrm{~F}$ & 6.87 & & 1 \\
\hline pailful & 1591 & 4.27 & $\mathbf{M}$ & 5.30 & $\mathrm{~F}$ & 3.80 & $\mathbf{F}$ & 5.20 & & 1 \\
\hline pallet & 1330 & 6.00 & & 5.50 & $\mathrm{~F}$ & 4.40 & . & 5.90 & M & 1 \\
\hline palmy & 1602 & 3.73 & & 4.27 & $\mathrm{~F}$ & 3.60 & . & 4.50 & $\mathbf{M}$ & 0 \\
\hline paned & 1555 & 3.60 & & 3.94 & & 3.70 & . & 3.93 & $\mathbf{M}$ & 0 \\
\hline papered & 1599 & 4.37 & & 4.77 & $\mathbf{F}$ & 4.14 & . & 5.47 & & 1 \\
\hline papillary & 1667 & 3.60 & & 2.23 & $\mathrm{~F}$ & 3.93 & . & 3.00 & $\mathbf{M}$ & 1 \\
\hline paraphragm & 1877 & 3.90 & $\mathbf{M}$ & 2.63 & $\mathbf{M}$ & 3.74 & 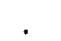 & 2.03 & $\mathbf{M}$ & 0 \\
\hline parch & 1398 & 4.50 & $\mathbf{M}$ & 4.23 & . & 2.57 & & 5.43 & $\mathbf{M}$ & 1 \\
\hline parkish & 1813 & 2.93 & & 2.84 & & 3.83 & M & 2.87 & M & 1 \\
\hline partiality & 1422 & 2.63 & & 3.27 & $F$ & 3.83 & $\mathbf{M}$ & 5.50 & & 1 \\
\hline partyism & 1842 & 2.77 & & 3.93 & $\mathbf{M}$ & 3.84 & . & 3.34 & $\mathbf{M}$ & 0 \\
\hline pass & 1225 & 3.67 & $\mathbf{M}$ & 6.20 & & 5.10 & & 6.94 & & 1 \\
\hline pat-a-cake & 1874 & 4.40 & $\mathrm{~F}$ & 5.67 & & 4.97 & $\mathbf{F}$ & 5.73 & F & 0 \\
\hline patrial & 1629 & 2.87 & & 3.20 & $\mathrm{~F}$ & 3.90 & & 3.70 & & 0 \\
\hline patterned & 1797 & 3.53 & & 5.30 & $\mathrm{~F}$ & 4.70 & $\mathbf{F}$ & 6.50 & & 1 \\
\hline peahen & 1377 & 3.40 & $\mathbf{M}$ & 1.77 & $\mathbf{M}$ & 3.47 & $\mathbf{F}$ & 1.97 & $\cdot$ & 0 \\
\hline pebble & 1290 & 6.37 & & 6.50 & $\mathrm{~F}$ & 4.67 & $\mathrm{~F}$ & 6.80 & & 1 \\
\hline pedestrianism & 1809 & 3.27 & $\mathbf{M}$ & 5.03 & $\mathrm{~F}$ & 4.67 & . & 5.17 & & 0 \\
\hline
\end{tabular}


APPENDIX (Continued)

\begin{tabular}{|c|c|c|c|c|c|c|c|c|c|c|}
\hline WORD & DOE & CONC & & IMAG & & GOOD & & FAM & & REQ \\
\hline pemmican & 1801 & 4.30 & $\mathbf{M}$ & 3.17 & $\mathrm{~F}$ & 3.67 & - & 2.80 & $\mathbf{M}$ & 1 \\
\hline pendule & 1578 & 4.27 & $\mathbf{F}$ & 2.63 & & 3.77 & & 3.04 & & 0 \\
\hline pennyweight & 1000 & 4.73 & . & 4.80 & $\mathrm{~F}$ & 4.10 & $\mathbf{F}$ & 4.13 & & 0 \\
\hline perceived & 1400 & 2.43 & & 3.90 & F & 4.67 & & 6.87 & & 1 \\
\hline perduring & 1501 & 2.73 & $\mathbf{F}$ & 2.07 & $\mathrm{~F}$ & 3.63 & $\mathbf{M}$ & 3.00 & $\mathbf{M}$ & 0 \\
\hline peri & 1777 & 2.57 & . & 2.47 & & 3.80 & . & 2.24 & & 1 \\
\hline pernickety & 1808 & 2.03 & . & 2.83 & $\mathrm{~F}$ & 2.97 & . & 2.57 & $\mathbf{M}$ & 0 \\
\hline personalist & 1876 & 2.90 & . & 3.40 & $\mathrm{~F}$ & 4.33 & 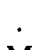 & 4.67 & & 0 \\
\hline persuasiveness & 1611 & 2.43 & & 4.17 & F & 4.74 & $\mathbf{M}$ & 6.24 & & 0 \\
\hline pettifog & 1611 & 3.93 & $\mathbf{M}$ & 2.43 & $\mathbf{M}$ & 3.67 & $\mathrm{~F}$ & 2.20 & $\mathbf{M}$ & 0 \\
\hline phase & 1812 & 2.77 & & 4.56 & . & 4.03 & . & 6.94 & & 1 \\
\hline philistia & 1535 & 2.60 & $\mathbf{M}$ & 1.53 & & 3.27 & . & 1.57 & $\mathbf{M}$ & 0 \\
\hline phosphorolysis & 1937 & 3.57 & . & 1.97 & $\mathbf{M}$ & 3.50 & . & 2.07 & $\mathrm{~F}$ & 0 \\
\hline photoscopic & 1872 & 3.67 & . & 3.67 & $\mathbf{F}$ & 4.87 & . & 3.64 & & 0 \\
\hline phylogenically & 1975 & 2.47 & . & 1.77 & . & 3.20 & & 2.80 & $\mathbf{M}$ & 0 \\
\hline pick & 1300 & 4.63 & $\mathbf{M}$ & 6.27 & . & 4.47 & F & 7.00 & . & 1 \\
\hline pie & 1250 & 6.40 & $\mathrm{~F}$ & 6.77 & . & 5.20 & . & 6.97 & . & 1 \\
\hline pied & 1382 & 3.47 & . & 3.23 & & 3.23 & & 3.74 & $\mathbf{F}$ & 1 \\
\hline piling & 1358 & 4.20 & . & 5.47 & F & 3.70 & & 6.07 & $\mathbf{M}$ & 1 \\
\hline pillowy & 1798 & 3.87 & . & 5.33 & & 5.27 & $\mathrm{~F}$ & 5.03 & . & 0 \\
\hline pin & 1100 & 6.70 & . & 6.54 & F & 3.67 & & 7.00 & . & 1 \\
\hline ping & 897 & 4.33 & . & 4.77 & . & 4.33 & $\mathrm{~F}$ & 5.24 & . & 1 \\
\hline Pirandellian & 1927 & 4.13 & . & 1.67 & . & 3.40 & & 1.50 & $\mathbf{M}$ & 0 \\
\hline piratical & 1565 & 2.43 & . & 2.80 & $\mathbf{M}$ & 3.43 & $\mathbf{M}$ & 2.54 & . & 0 \\
\hline pivot & 1611 & 4.57 & & 5.40 & . & 4.73 & . & 6.70 & . & 1 \\
\hline plancher & 1400 & 3.80 & $\mathrm{~F}$ & 2.37 & . & 3.23 & . & 2.10 & & $\mathbf{0}$ \\
\hline plane & 1320 & 6.47 & & 6.50 & . & 5.73 & . & 7.00 & . & 1 \\
\hline plate-layer & 1836 & 4.63 & & 4.03 & . & 3.93 & & 3.40 & . & $\mathbf{0}$ \\
\hline platoon & 1637 & 5.50 & $\mathbf{M}$ & 6.00 & . & 3.63 & $\mathbf{M}$ & 6.93 & . & 1 \\
\hline pleasure-ground & 1768 & 3.37 & . & 4.40 & & 6.10 & & 4.27 & & 0 \\
\hline plough-gate & 1565 & 5.20 & . & 3.67 & F & 3.47 & $\mathbf{F}$ & 3.07 & & 0 \\
\hline plugged & 1872 & 4.50 & . & 5.70 & $\mathrm{~F}$ & 3.50 & & 6.47 & $\mathrm{~F}$ & 1 \\
\hline plutonic & 1796 & 2.83 & & 4.00 & & 4.20 & $\mathbf{M}$ & 5.10 & . & 1 \\
\hline Poilite & 1903 & 3.73 & $\mathrm{~F}$ & 2.13 & & 3.93 & . & 1.97 & . & 0 \\
\hline point & 1225 & 5.30 & $\mathbf{M}$ & 6.30 & $\mathrm{~F}$ & 4.57 & & 6.77 & . & 1 \\
\hline policed & 1591 & 4.00 & . & 5.10 & & 3.97 & $\mathbf{M}$ & 6.37 & . & 1 \\
\hline politic & 1420 & 2.73 & . & 4.60 & $F$ & 3.43 & $\mathbf{M}$ & 6.10 & F & 1 \\
\hline polymeric & 1833 & 2.67 & . & 2.03 & . & 3.50 & . & 2.34 & $\mathbf{M}$ & 1 \\
\hline polysomic & 1932 & 3.20 & . & 1.90 & . & 3.73 & & 1.97 & $\mathbf{M}$ & 0 \\
\hline pool & 897 & 6.67 & . & 6.80 & & 5.93 & $\mathbf{F}$ & 6.97 & & 1 \\
\hline pop-shop & 1772 & 6.10 & & 5.94 & & 4.83 & $\mathrm{~F}$ & 5.83 & $\mathbf{M}$ & 0 \\
\hline porphyrite & 1577 & 3.90 & $\mathbf{M}$ & 1.47 & $\mathbf{M}$ & 3.53 & $\mathbf{M}$ & 1.47 & & 0 \\
\hline posological & 1803 & 2.53 & . & 1.43 & $\mathbf{M}$ & 3.13 & . & 1.83 & $\mathbf{M}$ & 0 \\
\hline postalize & 1893 & 2.90 & & 3.17 & $\mathbf{F}$ & 3.80 & . & 3.23 & & 0 \\
\hline postorbital & 1835 & 2.90 & $\mathbf{F}$ & 2.43 & $\mathbf{M}$ & 3.44 & . & 2.80 & $\mathbf{M}$ & 0 \\
\hline pottiness & 1933 & 2.87 & . & 3.40 & F & 2.97 & . & 3.14 & . & 0 \\
\hline potwaller & 1701 & 4.20 & & 2.30 & $\mathbf{F}$ & 3.43 & & 2.20 & & 1 \\
\hline practical & 1570 & 2.70 & $\mathbf{M}$ & 3.83 & . & 5.53 & $\mathbf{M}$ & 6.94 & & 1 \\
\hline praying & 1303 & 4.13 & $\mathbf{F}$ & 6.54 & . & 5.33 & & 6.97 & . & 1 \\
\hline preachify & 1775 & 2.63 & & 2.90 & . & 3.57 & $\mathrm{~F}$ & 3.10 & . & 0 \\
\hline predetermine & 1625 & 2.37 & $\mathbf{M}$ & 3.23 & $\mathrm{~F}$ & 4.57 & & 6.10 & & $\mathbf{0}$ \\
\hline preliterate & 1925 & 2.50 & & 2.73 & $\mathbf{F}$ & 3.87 & $\mathbf{M}$ & 4.10 & $\mathbf{M}$ & 1 \\
\hline premeridian & 1858 & 3.23 & $\mathrm{~F}$ & 2.50 & $\mathbf{M}$ & 3.53 & & 2.74 & & 0 \\
\hline presensation & 1653 & 4.17 & $F$ & 4.47 & $\mathrm{~F}$ & 4.43 & $\mathrm{~F}$ & 4.50 & $\mathbf{M}$ & 0 \\
\hline pressurized & 1938 & 3.87 & . & 5.17 & & 3.40 & & 6.07 & . & 1 \\
\hline prestigious & 1546 & 2.57 & . & 5.17 & F & 5.23 & $\mathbf{M}$ & 6.70 & . & 1 \\
\hline price & 1225 & 4.37 & . & 5.93 & $\mathbf{F}$ & 4.10 & $\cdot$ & 7.00 & & 1 \\
\hline primeval & 1653 & 3.00 & . & 3.47 & . & 3.57 & . & 5.07 & . & 1 \\
\hline printed & 1483 & 4.50 & . & 6.13 & . & 4.83 & & 6.94 & . & 1 \\
\hline prismatoidal & 1821 & 2.90 & . & 1.83 & . & 3.37 & & 1.70 & & 0 \\
\hline procedure & 1611 & 3.43 & . & 4.73 & . & 4.56 & $F$ & 6.93 & & 1 \\
\hline
\end{tabular}


APPENDIX (Continued)

\begin{tabular}{|c|c|c|c|c|c|c|c|c|c|c|}
\hline WORD & DOE & CONC & & IMAG & & GOOD & & FAM & & FREQ \\
\hline professing & 1502 & 2.77 & . & 4.63 & $\mathrm{~F}$ & 4.90 & & 6.07 & $\cdot$ & 1 \\
\hline profession & 1225 & 3.70 & . & 5.27 & $\mathbf{F}$ & 5.64 & & 6.97 & . & 1 \\
\hline prolificness & 1698 & 2.63 & . & 2.60 & & 3.83 & $\mathbf{M}$ & 3.03 & $\mathbf{M}$ & 0 \\
\hline propagand & 1795 & 3.27 & . & 3.07 & $\mathbf{F}$ & 2.87 & . & 3.94 & . & 0 \\
\hline propaganda & 1718 & 4.13 & $\mathbf{M}$ & 5.40 & $\mathbf{M}$ & 2.40 & . & 6.50 & $\mathbf{M}$ & 1 \\
\hline propensity & 1570 & 2.70 & . & 2.07 & $\mathbf{M}$ & 4.20 & & 4.20 & $\mathbf{M}$ & 1 \\
\hline proselyte & 1375 & 3.47 & $\mathbf{M}$ & 1.47 & $\mathbf{M}$ & 3.27 & $\mathbf{M}$ & 1.44 & . & 0 \\
\hline protolinguistic & 1964 & 2.80 & $\mathbf{F}$ & 1.70 & . & 3.60 & . & 2.50 & . & 0 \\
\hline protopathic & 1858 & 2.70 & . & 1.90 & . & 3.90 & . & 2.04 & . & 0 \\
\hline proxy & 1440 & 4.33 & $\mathbf{M}$ & 3.87 & $\cdot$ & 3.53 & & 5.27 & $\mathbf{M}$ & 1 \\
\hline psychedelicize & 1966 & 2.73 & $\mathbf{F}$ & 2.93 & $\mathbf{M}$ & 3.53 & $\mathbf{M}$ & 2.57 & $\mathbf{M}$ & 0 \\
\hline psychotic & 1890 & 3.37 & $\mathbf{M}$ & 5.03 & $\cdot$ & 1.87 & . & 6.63 & $\cdot$ & 1 \\
\hline pugilistic & 1789 & 2.83 & $\mathbf{M}$ & 2.10 & $\mathbf{M}$ & 2.80 & $\mathbf{M}$ & 2.50 & $\mathbf{M}$ & 1 \\
\hline pugnacious & 1642 & 2.53 & . & 2.07 & $\mathbf{M}$ & 2.70 & $\mathbf{M}$ & 3.70 & $\mathbf{M}$ & 1 \\
\hline punch & 1382 & 5.87 & . & 6.63 & . & 2.40 & . & 7.00 & . & 1 \\
\hline pureness & 1398 & 2.33 & . & 4.93 & $\mathrm{~F}$ & 6.00 & . & 6.53 & . & 0 \\
\hline Purex & 1956 & 4.03 & . & 2.77 & . & 4.06 & . & 2.70 & $\cdot$ & 0 \\
\hline purvey & 1290 & 2.80 & . & 1.93 & . & 3.70 & . & 2.80 & $\mathbf{M}$ & 0 \\
\hline putter & 1382 & 5.67 & . & 6.14 & $\mathrm{~F}$ & 4.50 & . & 6.30 & $\mathbf{M}$ & 1 \\
\hline Pyribenzamine & 1946 & 4.33 & . & 2.00 & . & 3.27 & . & 1.50 & . & 0 \\
\hline quad & 1820 & 3.70 & $\mathbf{M}$ & 4.90 & $\mathbf{M}$ & 4.13 & . & 5.50 & . & 0 \\
\hline quarrier & 1375 & 3.27 & . & 2.53 & . & 3.70 & . & 3.10 & $\mathbf{M}$ & 0 \\
\hline quarterly & 1450 & 3.00 & . & 5.27 & & 4.07 & . & 6.63 & . & 1 \\
\hline querier & 1672 & 3.53 & . & 2.54 & $\mathbf{M}$ & 3.43 & . & 2.44 & . & 0 \\
\hline quinquagint & 1843 & 2.57 & $\mathbf{M}$ & 1.47 & . & 2.87 & . & 1.47 & . & 0 \\
\hline quinquesect & 1697 & 2.80 & . & 1.40 & . & 3.50 & . & 1.44 & . & 0 \\
\hline race & 1300 & 4.90 & $\mathbf{M}$ & 6.57 & $\mathbf{F}$ & 5.07 & . & 7.00 & . & 1 \\
\hline raddle & 1523 & 3.87 & . & 4.13 & . & 3.70 & . & 3.80 & $\mathbf{F}$ & 0 \\
\hline rageful & 1580 & 3.50 & . & 5.77 & . & 2.07 & . & 5.17 & . & 0 \\
\hline raggy & 1100 & 3.93 & . & 5.60 & . & 2.83 & . & 4.83 & . & 0 \\
\hline rame & 1470 & 3.10 & . & 1.70 & . & 3.20 & . & 2.07 & $\mathbf{F}$ & 0 \\
\hline randomization & 1926 & 2.73 & $\mathbf{F}$ & 3.80 & . & 3.93 & $\mathbf{F}$ & 5.40 & $\mathbf{M}$ & 1 \\
\hline rape & 1086 & 5.57 & . & 6.37 & . & 1.10 & . & 7.00 & . & 1 \\
\hline rationalizing & 1865 & 2.43 & . & 4.10 & . & 4.64 & . & 6.50 & . & $\mathbf{0}$ \\
\hline ratter & 1834 & 3.57 & $\mathbf{M}$ & 3.27 & $\mathbf{F}$ & 3.00 & . & 3.37 & - & 0 \\
\hline reach-me-down & 1862 & 2.77 & . & 3.77 & $\mathbf{F}$ & 3.57 & . & 3.07 & $\mathbf{F}$ & 0 \\
\hline ready-to-wear & 1895 & 3.50 & $\mathbf{M}$ & 5.50 & $\mathrm{~F}$ & 5.00 & $\mathbf{F}$ & 6.13 & . & 0 \\
\hline reason & 1225 & 2.67 & $\mathbf{M}$ & 4.20 & $\mathbf{F}$ & 5.20 & . & 7.00 & . & 1 \\
\hline recapitulation & 1388 & 2.87 & $\mathbf{F}$ & 2.83 & . & 3.64 & $\mathbf{F}$ & 4.70 & $\mathbf{M}$ & 1 \\
\hline receiver & 1330 & 5.20 & $\mathbf{M}$ & 6.20 & . & 4.87 & , & 6.94 & • & 1 \\
\hline recoinage & 1622 & 2.67 & $\mathbf{M}$ & 2.97 & $\mathbf{F}$ & 3.60 & . & 3.27 & . & 0 \\
\hline recombinational & 1959 & 2.33 & . & 2.57 & . & 4.20 & . & 3.00 & $\mathbf{F}$ & 0 \\
\hline recrudescence & 1721 & 2.73 & . & 1.90 & $\mathbf{M}$ & 2.97 & $\mathbf{M}$ & 2.20 & . & 0 \\
\hline reddle & 1727 & 3.13 & . & 1.87 & $\mathbf{M}$ & 3.73 & . & 1.90 & $\mathbf{M}$ & 0 \\
\hline Redemptorist & 1835 & 4.57 & . & 2.17 & $\mathbf{M}$ & 3.50 & . & 1.90 & $\mathbf{M}$ & 0 \\
\hline redundancy & 1601 & 2.37 & . & 3.94 & . & 2.50 & . & 6.00 & . & 1 \\
\hline refind & 1621 & 2.87 & . & 3.97 & . & 4.90 & $\mathbf{F}$ & 4.67 & $\mathbf{F}$ & 0 \\
\hline reflux & 1430 & 3.47 & $\mathbf{M}$ & 3.13 & $\dot{ }$ & 3.53 & $\mathbf{M}$ & 3.03 & $\mathbf{M}$ & 0 \\
\hline refutal & 1605 & 2.70 & $\mathbf{F}$ & 3.24 & $\mathbf{F}$ & 3.10 & $\mathbf{M}$ & 3.97 & $\mathrm{~F}$ & 0 \\
\hline regurgitant & 1866 & 4.40 & . & 3.93 & . & 2.40 & . & 4.14 & $\mathbf{F}$ & 0 \\
\hline relativization & 1921 & 2.70 & $\cdot$ & 2.73 & . & 4.17 & . & 3.67 & $\mathbf{F}$ & 0 \\
\hline releasee & 1744 & 5.03 & $\mathbf{M}$ & 4.27 & . & 4.30 & . & 4.50 & . & 0 \\
\hline remeet & 1644 & 3.00 & . & 3.17 & $\mathbf{F}$ & 4.73 & . & 3.47 & F & 0 \\
\hline rendezvousing & 1679 & 3.17 & . & 4.23 & F & 5.06 & . & 4.64 & $\mathbf{F}$ & 1 \\
\hline repentable & 1571 & 2.37 & . & 3.24 & . & 4.37 & $\mathbf{M}$ & 4.74 & $\mathbf{F}$ & 0 \\
\hline replete & 1382 & 2.67 & . & 2.63 & . & 3.70 & & 4.44 & $\mathbf{M}$ & 1 \\
\hline repristination & 1838 & 2.73 & . & 1.80 & . & 3.67 & $\mathbf{M}$ & 2.07 & . & 0 \\
\hline resect & 1545 & 2.77 & . & 2.20 & . & 3.73 & . & 3.14 & . & 0 \\
\hline resolve & 1374 & 2.67 & $\mathbf{M}$ & 4.13 & . & 5.60 & . & 6.80 & & 1 \\
\hline resonance & 1491 & 3.47 & . & 2.90 & $\mathbf{M}$ & 4.47 & . & 5.10 & $\mathbf{M}$ & 1 \\
\hline rester & 1435 & 3.50 & $\mathbf{F}$ & 3.47 & $\mathbf{F}$ & 4.20 & . & 4.34 & $\mathrm{~F}$ & 0 \\
\hline
\end{tabular}


APPENDIX (Continued)

\begin{tabular}{|c|c|c|c|c|c|c|c|c|c|c|}
\hline WORD & DOE & CONC & & IMAG & & GOOD & & FAM & & FREQ \\
\hline retiform & 1691 & 2.83 & . & 1.60 & $\mathbf{M}$ & 3.64 & & 1.94 & & 0 \\
\hline return-ticket & 1847 & 5.90 & . & 6.13 & & 5.27 & & 6.67 & F & 0 \\
\hline returnable & 1425 & 2.97 & & 5.00 & $\mathrm{~F}$ & 5.20 & & 6.70 & & 1 \\
\hline revive & 1430 & 3.43 & $\mathbf{M}$ & 5.44 & $\mathbf{F}$ & 6.23 & & 6.80 & & 1 \\
\hline rhomboidal & 1658 & 3.07 & $\mathbf{M}$ & 2.33 & $\mathbf{M}$ & 3.63 & $\mathbf{M}$ & 2.07 & $\mathbf{M}$ & 0 \\
\hline rhyolite & 1866 & 3.57 & $\mathbf{M}$ & 1.40 & & 3.33 & & 1.50 & $\mathbf{M}$ & 1 \\
\hline ride & 888 & 4.53 & & 6.27 & $\mathrm{~F}$ & 5.40 & & 7.00 & & 1 \\
\hline right & 825 & 3.30 & $\mathbf{M}$ & 5.43 & . & 5.84 & & 7.00 & & 1 \\
\hline rigidize & 1948 & 2.90 & . & 3.27 & . & 3.00 & $\mathbf{M}$ & 3.70 & & 0 \\
\hline ringlet & 1555 & 5.57 & & 4.84 & $\mathbf{F}$ & 4.64 & $\mathrm{~F}$ & 5.77 & & 0 \\
\hline rivalship & 1632 & 3.10 & . & 4.53 & $\mathbf{F}$ & 3.40 & $\mathbf{M}$ & 4.57 & & 0 \\
\hline river & 1297 & 6.47 & . & 6.77 & . & 5.83 & & 7.00 & . & 1 \\
\hline roche & 1250 & 3.70 & $\mathbf{M}$ & 3.17 & & 3.00 & $\mathbf{M}$ & 2.47 & . & 0 \\
\hline rodded & 1562 & 3.33 & . & 3.47 & $\mathrm{~F}$ & 3.00 & . & 3.03 & . & 0 \\
\hline rollable & 1729 & 3.40 & & 5.20 & & 4.63 & $F$ & 4.70 & & 0 \\
\hline rooibok & 1824 & 3.17 & . & 1.37 & $\mathbf{M}$ & 3.53 & & 1.40 & $\mathbf{M}$ & 0 \\
\hline roominess & 1840 & 3.53 & & 5.47 & & 5.54 & $\mathrm{~F}$ & 5.83 & & 0 \\
\hline rosily & 1809 & 2.63 & & 4.23 & $\mathbf{F}$ & 5.50 & & 4.23 & $F$ & 1 \\
\hline Rouen & 1728 & 4.17 & $\mathbf{M}$ & 1.97 & $\mathbf{M}$ & 3.13 & & 1.57 & . & I \\
\hline round & 1000 & 5.07 & . & 6.47 & . & 4.80 & $F$ & 7.00 & & 1 \\
\hline rovingly & 1601 & 2.67 & . & 3.54 & . & 3.87 & . & 3.34 & $\mathbf{M}$ & 0 \\
\hline rubbish & 1392 & 5.73 & & 5.83 & $\mathbf{F}$ & 2.30 & . & 6.80 & & 1 \\
\hline ruddle & 1538 & 2.67 & $\mathbf{F}$ & 2.57 & . & 3.33 & . & 2.90 & . & 0 \\
\hline ruling & 1225 & 3.47 & & 4.90 & . & 4.00 & . & 6.90 & . & 1 \\
\hline run & 725 & 4.63 & $\mathrm{~F}$ & 6.47 & . & 5.50 & & 7.00 & . & 1 \\
\hline Runge-Kutta & 1930 & 4.27 & $\mathbf{M}$ & 1.60 & . & 3.20 & & 1.20 & & 0 \\
\hline rushy & 1382 & 3.03 & & 4.53 & & 3.26 & & 4.77 & $\mathbf{M}$ & 0 \\
\hline rust & 725 & 6.23 & & 6.54 & $\mathbf{F}$ & 2.27 & $\mathrm{~F}$ & 6.97 & & 1 \\
\hline Saadian & 1899 & 4.20 & $\mathbf{M}$ & 1.63 & $\mathbf{M}$ & 2.90 & & 1.33 & & 0 \\
\hline sack-friar & 1400 & 4.17 & & 2.70 & $\mathbf{M}$ & 3.13 & & 2.07 & $\mathbf{M}$ & 0 \\
\hline sagamore & 1613 & 3.40 & $\mathbf{M}$ & 1.87 & . & 3.54 & $F$ & 1.80 & . & 0 \\
\hline Sahli & 1906 & 4.63 & $\mathbf{M}$ & 1.97 & $\mathbf{M}$ & 3.30 & $\mathbf{M}$ & 1.20 & & 0 \\
\hline sallowness & 1722 & 3.57 & & 3.44 & $\mathbf{F}$ & 2.77 & . & 3.60 & $\mathrm{~F}$ & 0 \\
\hline same & 1200 & 2.90 & $\mathbf{M}$ & 4.17 & $\mathrm{~F}$ & 4.30 & . & 7.00 & . & 1 \\
\hline samfu & 1955 & 2.47 & & 1.50 & $\mathbf{M}$ & 3.54 & . & 1.47 & & 0 \\
\hline Sanhedrim & 1588 & 4.37 & & 1.57 & & 3.00 & . & 1.77 & $\mathbf{M}$ & 0 \\
\hline sargassum & 1905 & 3.57 & $\mathbf{M}$ & 1.87 & $\mathbf{M}$ & 2.76 & . & 2.07 & $\mathbf{M}$ & 0 \\
\hline satellite & 1548 & 6.40 & . & 6.37 & $\mathbf{F}$ & 5.14 & . & 6.80 & . & 1 \\
\hline save & 1225 & 3.20 & . & 5.33 & . & 5.74 & & 7.00 & & 1 \\
\hline scaffold & 1349 & 6.20 & & 5.90 & & 4.07 & $\mathbf{M}$ & 6.44 & $\mathbf{M}$ & 1 \\
\hline scale & 1205 & 5.40 & $\mathbf{M}$ & 6.37 & $\mathrm{~F}$ & 4.37 & . & 7.00 & & 1 \\
\hline scape & 1275 & 3.67 & $\mathbf{M}$ & 3.70 & & 3.43 & . & 5.44 & & 1 \\
\hline sceptic & 1575 & 3.77 & $\mathbf{M}$ & 4.87 & $\mathbf{F}$ & 2.97 & & 6.23 & $\mathbf{M}$ & 0 \\
\hline schmooze & 1897 & 3.10 & & 2.33 & & 3.77 & $\mathbf{F}$ & 2.30 & $\mathbf{M}$ & 0 \\
\hline scientific & 1589 & 2.53 & $\mathbf{M}$ & 4.97 & & 4.80 & . & 6.83 & . & 1 \\
\hline scorify & 1754 & 2.73 & $\mathbf{F}$ & 2.87 & $\mathbf{F}$ & 3.13 & & 2.00 & & 0 \\
\hline Scot & 900 & 5.13 & $\mathbf{M}$ & 5.00 & $\mathbf{F}$ & 4.37 & $\mathbf{F}$ & 5.50 & $\mathbf{M}$ & 1 \\
\hline scrape & 1000 & 4.17 & . & 6.30 & & 2.40 & & 6.83 & & 1 \\
\hline scripturally & 1679 & 2.93 & & 3.90 & $F$ & 4.43 & $\mathbf{M}$ & 5.40 & $\mathbf{F}$ & 0 \\
\hline scrounge & 1909 & 3.27 & $\mathbf{F}$ & 5.03 & $F$ & 2.40 & . & 6.20 & . & 0 \\
\hline scutellation & 1872 & 2.37 & & 1.50 & & 2.90 & . & 1.64 & & 0 \\
\hline sealskin & 1325 & 6.30 & $\mathbf{M}$ & 6.40 & & 3.20 & . & 6.57 & & 1 \\
\hline secernent & 1796 & 3.10 & & 1.67 & $\mathbf{M}$ & 3.37 & & 1.80 & & 0 \\
\hline second-guess & 1941 & 2.83 & $\mathbf{M}$ & 4.73 & . & 3.60 & $\mathbf{F}$ & 6.27 & & 0 \\
\hline securely & 1588 & 2.57 & & 4.90 & $\mathrm{~F}$ & 5.93 & & 6.67 & & 1 \\
\hline seel & 1500 & 3.27 & $\mathbf{M}$ & 3.74 & $\mathrm{~F}$ & 4.03 & $\mathbf{M}$ & 3.37 & $\mathbf{F}$ & 0 \\
\hline self & 888 & 4.53 & $\mathbf{M}$ & 6.24 & $\mathrm{~F}$ & 5.70 & . & 7.00 & & 1 \\
\hline self-actualization & 1939 & 2.67 & $\mathrm{~F}$ & 3.33 & $F$ & 5.90 & & 6.20 & $\mathbf{M}$ & 0 \\
\hline semblable & 1374 & 2.67 & . & 2.17 & & 3.40 & & 2.17 & & 0 \\
\hline seneschalty & 1577 & 2.63 & . & 1.57 & & 3.40 & $\mathbf{M}$ & 1.50 & & 0 \\
\hline sense & 1400 & 3.53 & . & 4.77 & $\mathbf{F}$ & 5.47 & . & 6.97 & & 1 \\
\hline
\end{tabular}


APPENDIX (Continued)

\begin{tabular}{|c|c|c|c|c|c|c|c|c|c|c|}
\hline WORD & DOE & CONC & & IMAG & & GOOD & & FAM & & FREQ \\
\hline separately & 1552 & 2.73 & - & 4.83 & $\mathbf{F}$ & 3.76 & & 6.77 & . & 1 \\
\hline sergeant & 1200 & 5.00 & . & 5.70 & $\mathrm{~F}$ & 4.00 & & 6.13 & . & 1 \\
\hline serosa & 1890 & 3.43 & & 1.74 & $\mathbf{M}$ & 3.37 & $\mathbf{M}$ & 1.57 & . & 0 \\
\hline service & 1100 & 3.70 & $\mathbf{M}$ & 5.33 & $\mathrm{~F}$ & 4.60 & $\mathbf{M}$ & 6.90 & . & 1 \\
\hline set & 725 & 4.17 & . & 5.40 & $\mathrm{~F}$ & 4.20 & $\mathrm{~F}$ & 7.00 & . & 1 \\
\hline Seto & 1881 & 3.50 & 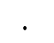 & 1.70 & $\mathbf{M}$ & 2.93 & . & 1.57 & . & 0 \\
\hline settle & 897 & 3.30 & $\mathbf{M}$ & 5.43 & . & 5.40 & . & 6.90 & & 1 \\
\hline sexennial & 1646 & 2.90 & . & 2.40 & $\mathbf{M}$ & 4.20 & . & 2.37 & $\mathbf{M}$ & 0 \\
\hline Shah & 1564 & 4.80 & $\mathbf{M}$ & 3.37 & M & 2.60 & . & 3.97 & $\mathbf{M}$ & 1 \\
\hline shamelessly & 897 & 2.77 & $\mathbf{M}$ & 4.50 & $\mathbf{F}$ & 3.83 & 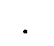 & 6.54 & . & 1 \\
\hline shape-up & 1940 & 3.37 & . & 5.60 & $\mathbf{F}$ & 4.90 & $\mathbf{M}$ & 6.64 & . & 1 \\
\hline shave & 725 & 4.90 & . & 6.60 & . & 4.37 & . & 7.00 & . & 1 \\
\hline sheep & 825 & 6.67 & . & 6.70 & $\cdot$ & 4.90 & $\mathbf{F}$ & 7.00 & . & 1 \\
\hline Shereefian & 1887 & 3.73 & . & 1.67 & $\mathbf{M}$ & 3.44 & . & 1.40 & . & 0 \\
\hline shimiyana & 1870 & 3.17 & . & 1.43 & $\mathbf{M}$ & 3.14 & . & 1.30 & . & 0 \\
\hline ship & 725 & 6.77 & . & 6.77 & $\dot{-}$ & 5.43 & . & 7.00 & . & 1 \\
\hline shoemaking & 1611 & 4.63 & . & 6.17 & $\mathrm{~F}$ & 4.64 & . & 6.37 & . & 0 \\
\hline short-change & 1903 & 3.70 & $\mathbf{M}$ & 4.73 & . & 2.27 & $\mathbf{F}$ & 6.10 & . & 0 \\
\hline shortcoming & 1680 & 2.83 & . & 4.17 & . & 2.93 & . & 6.74 & . & 1 \\
\hline show-box & 1748 & 5.40 & $\mathbf{M}$ & 4.33 & . & 4.73 & . & 4.04 & . & 0 \\
\hline shudder & 1310 & 5.10 & $F$ & 5.90 & . & 2.73 & . & 6.03 & . & 1 \\
\hline Sican & 1887 & 3.77 & $\mathbf{M}$ & 1.63 & $\mathbf{M}$ & 3.07 & . & 1.37 & . & 0 \\
\hline sickly & 1350 & 3.97 & $\mathrm{~F}$ & 5.80 & · & 1.57 & . & 6.60 & . & 1 \\
\hline sigmoiid & 1670 & 3.60 & . & 1.77 & $\mathbf{M}$ & 3.07 & $\mathbf{M}$ & 2.30 & . & 0 \\
\hline Silesian & 1645 & 4.47 & . & 2.03 & $\mathbf{M}$ & 3.37 & . & 2.03 & $\mathbf{M}$ & 0 \\
\hline silure & 1802 & 3.10 & . & 1.33 & $\mathbf{M}$ & 3.10 & . & 1.67 & $\mathbf{M}$ & 0 \\
\hline sindon & 1450 & 3.10 & . & 1.40 & $\mathbf{M}$ & 3.30 & . & 1.43 & $\mathbf{M}$ & 0 \\
\hline sink-stone & 1766 & 5.27 & . & 4.54 & $F$ & 3.43 & . & 3.40 & $\therefore$ & 0 \\
\hline sinsemilla & 1975 & 3.47 & . & 1.70 & $\mathbf{M}$ & 3.40 & . & 2.07 & $\mathbf{M}$ & 0 \\
\hline sitter & 1340 & 5.17 & $F$ & 5.84 & $F$ & 4.27 & $\mathrm{~F}$ & 6.27 & $\mathbf{F}$ & 1 \\
\hline skew & 1278 & 4.13 & . & 3.90 & $\mathbf{M}$ & 2.37 & . & 4.60 & $\mathbf{M}$ & 1 \\
\hline skimmer & 1387 & 4.97 & $\mathbf{M}$ & 5.50 & . & 4.37 & . & 5.70 & $\mathbf{F}$ & 0 \\
\hline skulking & 1297 & 3.17 & . & 4.00 & . & 2.73 & $\mathbf{M}$ & 4.14 & $\mathbf{M}$ & 1 \\
\hline sky & 1220 & 6.47 & . & 6.84 & . & 6.37 & . & 7.00 & . & 1 \\
\hline slate & 1799 & 5.87 & . & 6.07 & $\mathbf{F}$ & 4.37 & . & 6.73 & . & 1 \\
\hline slaughter & 1300 & 5.03 & $\mathrm{~F}$ & 6.37 & . & 1.57 & . & 6.94 & . & 1 \\
\hline sleight & 893 & 3.40 & . & 3.54 & $\mathrm{~F}$ & 3.73 & $\mathbf{F}$ & 4.47 & . & 1 \\
\hline slip & 1000 & 5.30 & $\mathbf{M}$ & 6.40 & . & 3.03 & $\therefore$ & 7.00 & . & 1 \\
\hline sloshed & 1946 & 4.13 & $\cdot$ & 5.73 & . & 3.20 & $\mathbf{M}$ & 6.37 & & 1 \\
\hline slow-going & 1798 & 3.43 & . & 5.34 & . & 2.97 & $\mathbf{F}$ & 6.20 & $F$ & 0 \\
\hline smart & 893 & 3.10 & . & 5.37 & . & 6.00 & . & 7.00 & & 1 \\
\hline smoke-ball & 1753 & 5.63 & $\mathbf{M}$ & 6.14 & $\mathbf{F}$ & 2.50 & . & 3.80 & $\mathbf{M}$ & 0 \\
\hline smooth & 1050 & 4.53 & $\mathbf{M}$ & 6.47 & . & 5.67 & $\mathbf{F}$ & 6.97 & . & 1 \\
\hline snap & 1495 & 4.97 & . & 6.30 & . & 3.50 & $\mathrm{~F}$ & 6.84 & . & 1 \\
\hline snobbess & 1869 & 3.10 & $\mathrm{~F}$ & 5.00 & . & 2.00 & . & 3.53 & $\mathrm{~F}$ & 0 \\
\hline snowed & 1682 & 4.90 & . & 6.50 & . & 4.90 & $\mathrm{~F}$ & 6.70 & . & 1 \\
\hline so & 700 & 1.80 & $\mathbf{M}$ & 2.24 & . & 3.83 & . & 7.00 & . & 1 \\
\hline soffit & 1613 & 4.13 & . & 2.83 & $\mathbf{F}$ & 4.37 & . & 1.87 & $\mathbf{M}$ & 0 \\
\hline soft & 1000 & 4.30 & $\mathbf{F}$ & 6.33 & . & 6.20 & $\mathrm{~F}$ & 6.90 & . & 1 \\
\hline sole & 1000 & 5.70 & $\mathbf{M}$ & 5.80 & $\mathbf{F}$ & 4.50 & . & 6.77 & . & 1 \\
\hline somatologist & 1893 & 4.97 & $\mathbf{F}$ & 2.30 & $\mathbf{M}$ & 3.77 & . & 2.27 & $\mathbf{M}$ & 0 \\
\hline son & 825 & 6.00 & $\therefore$ & 6.70 & $\dot{ }$ & 5.87 & $\mathbf{M}$ & 7.00 & . & 1 \\
\hline soothe & 950 & 3.57 & $\mathbf{M}$ & 5.70 & $\mathbf{F}$ & 5.67 & $\mathrm{~F}$ & 6.60 & . & 1 \\
\hline sorry & 888 & 2.70 & - & 5.04 & $\mathrm{~F}$ & 4.30 & . & 7.00 & . & 1 \\
\hline sounding-line & 1336 & 3.83 & . & 3.26 & $\mathbf{F}$ & 3.97 & . & 4.20 & . & 0 \\
\hline southdown & 1787 & 2.80 & . & 3.77 & . & 4.47 & . & 3.97 & . & 0 \\
\hline sower & 1000 & 4.40 & $\mathbf{F}$ & 4.13 & $\cdot$ & 3.83 & $\mathrm{~F}$ & 4.50 & $\mathrm{~F}$ & 1 \\
\hline spare & 825 & 3.67 & $\mathbf{M}$ & 5.73 & . & 5.03 & . & 6.97 & . & 1 \\
\hline speak & 825 & 4.83 & $\mathbf{F}$ & 6.30 & $\mathbf{F}$ & 5.60 & . & 6.94 & . & 1 \\
\hline special & 1225 & 2.60 & $\therefore$ & 4.57 & . & 6.33 & . & 6.90 & . & 1 \\
\hline spectrograph & 1884 & 5.27 & $\mathbf{M}$ & 3.80 & . & 4.30 & $\mathbf{F}$ & 3.54 & $\mathbf{M}$ & 1 \\
\hline
\end{tabular}


APPENDIX (Continued)

\begin{tabular}{|c|c|c|c|c|c|c|c|c|c|c|}
\hline WORD & DOE & CONC & & IMAG & & GOOD & & FAM & & REQ \\
\hline spent & 1440 & 3.53 & $\mathbf{M}$ & 5.67 & $\mathrm{~F}$ & 3.73 & & 6.94 & & 1 \\
\hline spiky & 1578 & 4.50 & & 5.20 & F & 3.20 & $\mathbf{M}$ & 5.03 & F & 1 \\
\hline spin & 725 & 4.47 & & 6.23 & & 4.73 & $\mathrm{~F}$ & 6.93 & . & 1 \\
\hline spirit & 1250 & 3.97 & & 6.00 & & 5.87 & $\mathbf{F}$ & 7.00 & . & 1 \\
\hline splendour & 1450 & 3.30 & $\mathbf{M}$ & 5.10 & $\mathrm{~F}$ & 6.10 & $\mathbf{F}$ & 6.57 & & 1 \\
\hline spooler & 1554 & 3.93 & & 3.37 & & 4.00 & $\mathbf{F}$ & 3.24 & $\mathbf{M}$ & 0 \\
\hline sporadic & 1689 & 2.93 & $\mathbf{M}$ & 3.70 & $\mathbf{M}$ & 3.43 & & 4.87 & $\mathbf{M}$ & 1 \\
\hline spread & 1200 & 4.00 & $\mathbf{M}$ & 6.37 & & 4.80 & . & 6.97 & . & 1 \\
\hline sprouted & 1483 & 4.13 & & 5.83 & F & 5.20 & . & 6.57 & & 1 \\
\hline squamose & 1661 & 3.07 & . & 1.77 & & 3.43 & & 2.10 & $\mathbf{M}$ & 0 \\
\hline square & 1300 & 6.53 & . & 6.60 & . & 3.90 & $F$ & 7.00 & . & 1 \\
\hline squat & 1300 & 4.70 & . & 6.10 & . & 3.50 & & 6.67 & . & 1 \\
\hline squinny & 1605 & 2.87 & . & 2.50 & . & 3.00 & . & 2.03 & . & 0 \\
\hline stage & 1300 & 5.83 & . & 6.50 & . & 4.94 & . & 7.00 & . & 1 \\
\hline stall & 725 & 5.30 & & 6.00 & . & 2.87 & . & 6.87 & . & 1 \\
\hline stand & 825 & 5.13 & & 6.17 & & 5.03 & . & 7.00 & . & 1 \\
\hline standard & 1154 & 3.17 & $\mathbf{M}$ & 5.00 & $\mathbf{F}$ & 4.67 & . & 6.87 & . & 1 \\
\hline star & 825 & 6.37 & $\mathbf{M}$ & 6.87 & . & 6.24 & . & 7.00 & & 1 \\
\hline state & 1225 & 4.37 & & 5.84 & & 4.20 & & 7.00 & & 1 \\
\hline staveless & 1866 & 2.63 & $\mathbf{M}$ & 2.30 & $\mathbf{M}$ & 3.17 & . & 2.70 & & 0 \\
\hline steady & 1530 & 3.50 & & 5.30 & . & 5.34 & . & 6.94 & & 1 \\
\hline steed & 900 & 4.50 & $\mathbf{M}$ & 4.40 & . & 4.56 & . & 4.77 & $\mathbf{M}$ & 1 \\
\hline stenograph & 1821 & 5.53 & . & 4.40 & & 4.13 & . & 5.50 & $\mathbf{M}$ & 0 \\
\hline stevedore & 1788 & 4.17 & . & 2.37 & $\mathbf{M}$ & 3.53 & . & 2.27 & $\mathbf{M}$ & 1 \\
\hline stick & 888 & 6.57 & . & 6.70 & . & 3.93 & & 6.80 & & 1 \\
\hline stigmatic & 1594 & 2.73 & . & 3.00 & . & 3.27 & $\mathbf{M}$ & 4.30 & $\mathbf{M}$ & 0 \\
\hline stipulode & 1880 & 3.17 & & 1.64 & & 3.43 & . & 2.00 & $\mathbf{M}$ & 0 \\
\hline stockinet & 1824 & 3.57 & $\mathbf{F}$ & 2.77 & $\mathbf{F}$ & 3.64 & . & 2.27 & & 0 \\
\hline stooge & 1913 & 4.63 & . & 5.40 & $\mathbf{M}$ & 2.80 & & 6.50 & $\mathbf{M}$ & 0 \\
\hline stook & 1460 & 3.70 & . & 1.90 & . & 3.26 & $\mathbf{M}$ & 2.57 & . & 0 \\
\hline storm & 825 & 6.47 & & 6.84 & & 3.03 & . & 7.00 & 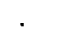 & 1 \\
\hline straight & 1300 & 4.47 & $\mathbf{M}$ & 6.40 & $\mathrm{~F}$ & 5.23 & . & 7.00 & & 1 \\
\hline strangle & 1300 & 4.53 & & 6.53 & . & 1.63 & . & 6.80 & & 1 \\
\hline streamline & 1918 & 4.20 & $\mathbf{M}$ & 5.90 & . & 5.30 & . & 6.17 & $\mathbf{M}$ & 1 \\
\hline street & 847 & 6.67 & & 6.50 & & 5.17 & . & 7.00 & . & 1 \\
\hline stridulant & 1843 & 2.90 & $\mathbf{F}$ & 1.90 & $\mathbf{M}$ & 3.33 & & 2.13 & . & 0 \\
\hline strip & 1200 & 4.40 & $\mathbf{M}$ & 6.54 & & 3.80 & $\mathbf{M}$ & 6.97 & . & 1 \\
\hline strong & 825 & 3.37 & . & 6.24 & & 5.97 & $\mathrm{~F}$ & 6.97 & . & 1 \\
\hline structural & 1835 & 3.57 & . & 5.27 & $\mathbf{M}$ & 5.34 & & 6.70 & . & 1 \\
\hline stuff & 1330 & 4.27 & . & 6.00 & $\mathbf{F}$ & 4.27 & $F$ & 7.00 & . & 1 \\
\hline styleless & 1796 & 2.60 & & 4.60 & $\mathrm{~F}$ & 2.97 & & 5.10 & . & 0 \\
\hline subduer & 1510 & 4.53 & $\mathrm{~F}$ & 3.63 & $\mathbf{M}$ & 3.20 & 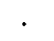 & 5.30 & . & 0 \\
\hline subjunctive & 1530 & 2.73 & - & 2.93 & $\mathrm{~F}$ & 3.27 & & 4.10 & & 0 \\
\hline subordinacy & 1627 & 2.53 & & 3.27 & . & 2.93 & . & 5.06 & & 0 \\
\hline substitutional & 1786 & 3.00 & & 4.13 & $\mathrm{~F}$ & 4.43 & . & 5.64 & & 0 \\
\hline succinated & 1698 & 2.63 & $\dot{0}$ & 1.90 & $\mathbf{M}$ & 3.76 & . & 2.20 & $\mathbf{M}$ & 0 \\
\hline suety & 1730 & 2.80 & $\mathbf{F}$ & 2.54 & & 3.43 & . & 1.90 & . & 0 \\
\hline suit & 1297 & 5.97 & & 6.60 & $F$ & 4.93 & . & 6.97 & & 1 \\
\hline summability & 1904 & 2.77 & $\mathbf{M}$ & 2.93 & . & 4.10 & . & 3.84 & . & 0 \\
\hline summer & 825 & 4.77 & . & 6.73 & & 6.37 & . & 6.94 & . & 1 \\
\hline sun-spot & 1818 & 5.50 & . & 5.70 & 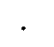 & 4.83 & . & 5.87 & $\mathbf{M}$ & 0 \\
\hline superjacent & 1610 & 3.37 & . & 2.17 & & 3.97 & . & 3.24 & . & 0 \\
\hline supply & 1374 & 3.67 & $\mathbf{M}$ & 4.97 & F & 4.80 & . & 6.84 & . & 1 \\
\hline supporting & 1413 & 3.27 & . & 5.00 & . & 5.60 & $F$ & 6.93 & . & 1 \\
\hline sure & 1330 & 2.77 & . & 3.90 & . & 5.47 & $\mathbf{F}$ & 7.00 & . & 1 \\
\hline surveillant & 1819 & 2.97 & $\mathrm{~F}$ & 3.50 & & 3.87 & $\mathbf{M}$ & 4.64 & 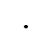 & 0 \\
\hline swad & 1460 & 3.30 & $\cdot$ & 2.67 & $\mathbf{F}$ & 3.20 & . & 2.37 & & 0 \\
\hline swatch & 1512 & 4.87 & & 5.07 & F & 4.63 & . & 6.24 & $\mathrm{~F}$ & 0 \\
\hline swearing & 1200 & 4.80 & $\mathrm{~F}$ & 5.80 & . & 2.93 & . & 6.97 & . & 1 \\
\hline
\end{tabular}


APPENDIX (Continued)

\begin{tabular}{|c|c|c|c|c|c|c|c|c|c|c|}
\hline WORD & DOE & CONC & & IMAG & & GOOD & & FAM & & FREQ \\
\hline sweet-brier & 1538 & 3.80 & $\mathrm{~F}$ & 3.27 & & 4.33 & r & 2.30 & & 0 \\
\hline swindling & 1527 & 3.23 & & 5.17 & & 2.30 & . & 6.04 & $\mathbf{M}$ & 1 \\
\hline swow & 1000 & 3.70 & $\mathrm{~F}$ & 3.47 & $\mathbf{M}$ & 4.00 & . & 2.47 & & 0 \\
\hline syanthesis & 1832 & 2.87 & $\mathbf{M}$ & 1.40 & . & 3.57 & . & 2.00 & $\mathbf{M}$ & 0 \\
\hline syllabic & 1728 & 3.13 & & 3.00 & $\mathbf{F}$ & 4.00 & . & 4.14 & $\mathbf{M}$ & 1 \\
\hline symphonist & 1656 & 5.77 & $\mathrm{~F}$ & 5.44 & . & 5.23 & . & 4.60 & . & 0 \\
\hline systemic & 1724 & 2.93 & & 3.90 & & 4.33 & . & 5.57 & & 1 \\
\hline tabriz & 1859 & 4.53 & $\mathbf{M}$ & 1.43 & $\mathbf{M}$ & 3.40 & & 1.44 & $\mathbf{M}$ & 1 \\
\hline tackle & 1250 & 5.40 & . & 6.30 & . & 4.03 & $\mathbf{M}$ & 6.84 & & 1 \\
\hline take & 1100 & 3.13 & . & 5.43 & . & 3.67 & $\mathbf{M}$ & 7.00 & . & 1 \\
\hline talk-back & 1933 & 3.43 & . & 5.47 & . & 2.44 & . & 6.07 & . & 0 \\
\hline tallying & 1440 & 3.37 & . & 5.24 & . & 4.23 & . & 5.57 & . & 0 \\
\hline taper & 897 & 4.40 & $\mathbf{M}$ & 5.10 & F & 4.07 & $\mathrm{~F}$ & 5.94 & . & 1 \\
\hline tarragona & 1888 & 4.57 & . & 2.17 & . & 3.53 & & 1.47 & . & 0 \\
\hline task & 1114 & 4.07 & . & 5.53 & . & 4.53 & $\mathrm{~F}$ & 6.87 & & 1 \\
\hline tea-board & 1748 & 5.70 & . & 4.57 & . & 4.17 & & 3.64 & & 0 \\
\hline teddy & 1907 & 6.13 & . & 5.93 & $\mathrm{~F}$ & 5.63 & F & 6.80 & . & 1 \\
\hline tele & 888 & 3.13 & . & 3.50 & & 4.23 & & 4.07 & . & 1 \\
\hline templed & 1610 & 3.93 & . & 3.77 & $F$ & 4.27 & $\mathbf{M}$ & 4.20 & . & 0 \\
\hline tenderizer & 1958 & 5.37 & . & 5.60 & $\mathbf{F}$ & 4.53 & & 6.67 & . & 1 \\
\hline tenseless & 1886 & 2.70 & & 4.54 & $\mathrm{~F}$ & 5.00 & $\mathrm{~F}$ & 4.97 & & 0 \\
\hline terraced & 1644 & 3.73 & $\mathbf{M}$ & 4.57 & $\mathrm{~F}$ & 4.47 & & 4.40 & $\mathbf{M}$ & 1 \\
\hline tetract & 1886 & 3.40 & $\mathbf{M}$ & 1.60 & . & 3.53 & $\mathbf{M}$ & 1.90 & $\mathbf{M}$ & 0 \\
\hline tetromino & 1954 & 3.60 & & 1.40 & . & 3.50 & . & 1.64 & . & 0 \\
\hline that & 825 & 2.07 & $\mathbf{M}$ & 3.07 & . & 3.83 & & 7.00 & & 1 \\
\hline theocracy & 1622 & 2.57 & & 1.87 & . & 3.47 & & 3.40 & $\mathbf{M}$ & 1 \\
\hline theromorph & 1887 & 3.73 & $\mathbf{M}$ & 1.97 & . & 3.50 & & 1.64 & $\mathbf{M}$ & 0 \\
\hline thin-skinned & 1598 & 4.00 & . & 5.77 & & 2.70 & $\mathbf{M}$ & 5.40 & . & 0 \\
\hline thing & 685 & 4.20 & . & 4.84 & & 4.57 & & 7.00 & . & 1 \\
\hline thorough & 800 & 2.53 & & 4.40 & $\mathbf{M}$ & 5.57 & & 6.93 & & 1 \\
\hline three-edged & 1398 & 4.80 & & 5.23 & . & 3.80 & $\mathrm{~F}$ & 5.37 & F & 0 \\
\hline throw & 888 & 4.43 & $\mathbf{F}$ & 6.40 & . & 4.30 & $\mathbf{M}$ & 7.00 & & 1 \\
\hline thrown & 1463 & 4.27 & $\mathrm{~F}$ & 6.07 & . & 3.50 & $\mathrm{~F}$ & 6.97 & & 1 \\
\hline thwack & 1530 & 3.17 & $\mathbf{M}$ & 4.30 & . & 2.64 & & 3.37 & $\mathbf{M}$ & 1 \\
\hline tie & 800 & 6.37 & & 6.53 & & 4.67 & $\mathrm{~F}$ & 6.94 & & 1 \\
\hline timbering & 1175 & 3.67 & $\mathbf{M}$ & 5.47 & $F$ & 3.87 & $\mathrm{~F}$ & 5.20 & F & 0 \\
\hline time & 893 & 3.13 & . & 5.87 & $F$ & 5.07 & . & 7.00 & & 1 \\
\hline tin-pot & 1772 & 5.87 & & 6.27 & . & 4.00 & . & 4.80 & $\mathbf{M}$ & 0 \\
\hline title & 950 & 4.67 & . & 5.84 & . & 4.87 & . & 7.00 & . & 1 \\
\hline to-day & 897 & 3.33 & . & 5.23 & $\mathbf{F}$ & 5.83 & . & 6.60 & & 1 \\
\hline togger & 1891 & 4.37 & & 2.07 & . & 3.84 & & 1.64 & $\mathbf{M}$ & 0 \\
\hline tomb & 1275 & 6.33 & $\mathbf{M}$ & 6.74 & . & 2.40 & $\mathbf{M}$ & 6.97 & . & 1 \\
\hline tooth & 725 & 6.60 & . & 6.80 & & 4.80 & . & 6.97 & . & 1 \\
\hline tor & 847 & 3.00 & . & 2.13 & $\mathbf{M}$ & 3.24 & . & 1.83 & & 1 \\
\hline tornadic & 1884 & 2.97 & & 3.27 & $\mathbf{M}$ & 2.63 & . & 2.37 & $\mathbf{M}$ & 0 \\
\hline touch & 1297 & 5.20 & $\mathbf{F}$ & 6.37 & $\mathrm{~F}$ & 6.17 & 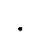 & 7.00 & & 1 \\
\hline townling & 1887 & 3.83 & & 2.77 & & 3.83 & . & 3.17 & $\mathbf{M}$ & 0 \\
\hline trace & 1300 & 3.83 & & 5.60 & $\mathrm{~F}$ & 4.50 & . & 6.90 & & 1 \\
\hline tradecraft & 1810 & 3.43 & & 3.74 & . & 4.70 & & 4.30 & $\mathrm{~F}$ & 0 \\
\hline tradition & 1380 & 3.23 & & 5.13 & $\mathrm{~F}$ & 5.40 & $\mathbf{M}$ & 6.90 & . & 1 \\
\hline tramp & 1388 & 5.57 & $\mathbf{M}$ & 6.47 & $F$ & 2.07 & . & 6.90 & & 1 \\
\hline trans-Siberian & 1896 & 4.30 & & 3.87 & $\mathbf{M}$ & 3.13 & . & 2.84 & $\mathbf{M}$ & 1 \\
\hline translatist & 1638 & 4.07 & F & 3.60 & $F$ & 4.31 & . & 3.47 & & 0 \\
\hline trap & 900 & 6.07 & F & 6.60 & 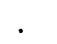 & 2.70 & . & 7.00 & & 1 \\
\hline treatise & 1374 & 3.90 & $\mathbf{M}$ & 2.97 & & 4.23 & 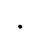 & 3.34 & $\mathbf{M}$ & 1 \\
\hline trestle-tree & 1625 & 5.40 & $\mathbf{M}$ & 3.54 & F & 4.53 & $\mathrm{~F}$ & 2.17 & & 0 \\
\hline tried & 1362 & 2.87 & $\mathbf{F}$ & 4.47 & . & 4.70 & & 6.97 & & 1 \\
\hline trig & 1200 & 3.47 & $\mathbf{M}$ & 4.23 & & 3.67 & & 3.74 & & 1 \\
\hline trip & 1305 & 4.83 & $\mathbf{M}$ & 6.60 & $\mathbf{F}$ & 6.27 & . & 6.97 & & 1 \\
\hline troll & 1377 & 5.53 & $\mathbf{M}$ & 5.37 & & 3.00 & - & 5.97 & & 1 \\
\hline
\end{tabular}


APPENDIX (Continued)

\begin{tabular}{|c|c|c|c|c|c|c|c|c|c|c|}
\hline WORD & DOE & CONC & & IMAG & & GOOD & & FAM & & REQ \\
\hline truck & 1225 & 6.77 & & 6.74 & $\mathbf{F}$ & 4.73 & & 7.00 & & 1 \\
\hline trunk & 1440 & 6.57 & & 6.60 & $\mathbf{F}$ & 4.54 & & 7.00 & & I \\
\hline truth & 893 & 2.83 & $\mathbf{M}$ & 4.94 & & 6.30 & & 6.97 & & 1 \\
\hline tuilyie & 1375 & 2.80 & & 1.40 & $\mathbf{M}$ & 3.20 & & 1.34 & $\mathbf{M}$ & 0 \\
\hline turf & 725 & 6.23 & & 6.04 & & 4.77 & & 6.83 & & 1 \\
\hline turn & 1000 & 4.23 & & 5.80 & $\mathbf{F}$ & 4.40 & & 7.00 & & 1 \\
\hline turnerite & 1823 & 3.70 & . & 1.73 & $\mathbf{M}$ & 3.43 & $\mathbf{M}$ & 1.83 & $\mathbf{M}$ & 0 \\
\hline twanger & 1598 & 3.90 & & 3.50 & $\mathbf{F}$ & 3.24 & . & 2.97 & & $\mathbf{0}$ \\
\hline twist & 1336 & 4.50 & F & 6.30 & & 4.23 & . & 6.93 & & 1 \\
\hline typology & 1845 & 3.20 & $\mathrm{~F}$ & 2.50 & . & 3.73 & . & 3.93 & $\mathbf{M}$ & 1 \\
\hline tyre & 1429 & 3.87 & $\mathbf{M}$ & 2.84 & . & 3.60 & . & 2.23 & $\mathbf{M}$ & 1 \\
\hline umbellated & 1676 & 2.33 & . & 2.20 & & 3.67 & . & 2.33 & $\mathbf{M}$ & 0 \\
\hline un-Greek & 1535 & 3.40 & . & 3.30 & $F$ & 3.30 & . & 3.10 & $\mathbf{M}$ & 0 \\
\hline unaccountableness & 1676 & 2.33 & & 3.30 & $\mathbf{F}$ & 2.63 & . & 4.97 & & 0 \\
\hline unbelieve & 1547 & 2.13 & $\mathbf{M}$ & 4.20 & . & 3.37 & . & 4.67 & . & 0 \\
\hline uncertified & 1535 & 2.70 & $\mathbf{M}$ & 4.20 & & 2.87 & & 6.54 & & 1 \\
\hline unchangeableness & 1548 & 2.60 & . & 3.50 & $\mathbf{F}$ & 2.67 & F & 4.97 & . & 0 \\
\hline uncontemplated & 1709 & 2.33 & . & 3.27 & $\mathbf{F}$ & 3.77 & . & 4.87 & & 0 \\
\hline under & 825 & 3.87 & . & 6.03 & & 3.43 & . & 7.00 & . & 1 \\
\hline underquote & 1891 & 2.60 & & 3.23 & $\mathrm{~F}$ & 2.60 & & 4.34 & & 0 \\
\hline undivulged & 1605 & 2.77 & $\mathbf{M}$ & 2.83 & & 3.60 & & 4.27 & $\mathbf{M}$ & 0 \\
\hline unfancied & 1655 & 2.43 & . & 3.70 & F & 3.10 & & 4.50 & & 0 \\
\hline unimprisoned & 1659 & 2.87 & . & 5.00 & $\mathbf{F}$ & 4.83 & $\mathbf{M}$ & 4.80 & & 0 \\
\hline unisex & 1968 & 3.50 & . & 5.10 & $\mathbf{F}$ & 4.23 & $\mathrm{~F}$ & 6.64 & & 0 \\
\hline unjustified & 1340 & 2.57 & . & 3.84 & & 2.50 & F & 6.74 & & 1 \\
\hline unmeaning & 1704 & 2.57 & . & 3.13 & $\mathbf{F}$ & 2.97 & . & 4.33 & & 0 \\
\hline unpersuadable & 1586 & 2.77 & . & 3.50 & $\mathbf{F}$ & 3.03 & . & 5.47 & & 0 \\
\hline unrefreshing & 1814 & 3.50 & $\mathbf{F}$ & 4.73 & $\mathbf{F}$ & 2.26 & . & 5.54 & $\mathbf{F}$ & $\mathbf{0}$ \\
\hline unseeable & 1395 & 2.73 & . & 3.73 & F & 2.77 & . & 4.80 & $\mathbf{M}$ & 0 \\
\hline unstamped & 1594 & 4.17 & $\mathbf{M}$ & 4.77 & $\mathbf{F}$ & 3.57 & . & 5.94 & $\mathbf{F}$ & $\mathbf{0}$ \\
\hline untirable & 1607 & 2.60 & $\mathbf{F}$ & 4.44 & $\mathbf{F}$ & 5.00 & $\mathrm{~F}$ & 4.54 & $\mathbf{M}$ & $\mathbf{0}$ \\
\hline unwhig & 1756 & 3.17 & $\mathbf{M}$ & 2.43 & & 3.34 & . & 2.20 & $\mathbf{M}$ & $\mathbf{0}$ \\
\hline up & 825 & 3.40 & $\mathbf{M}$ & 6.10 & $\mathbf{F}$ & 5.77 & . & 7.00 & & 1 \\
\hline up-put & 1689 & 2.73 & $\mathbf{M}$ & 3.00 & & 4.27 & & 3.37 & $F$ & $\mathbf{0}$ \\
\hline upswept & 1791 & 3.27 & $\mathbf{M}$ & 4.73 & $\mathbf{F}$ & 3.83 & F & 4.93 & . & 1 \\
\hline urgence & 1592 & 2.43 & & 3.90 & $\mathbf{F}$ & 4.14 & & 6.24 & & 0 \\
\hline usurer & 1290 & 4.27 & $\mathbf{M}$ & 2.83 & $\mathbf{M}$ & 3.17 & $\mathbf{M}$ & 2.80 & $\mathbf{M}$ & 1 \\
\hline vacuity & 1541 & 2.93 & . & 2.03 & $\mathbf{M}$ & 3.17 & & 2.30 & $\mathbf{M}$ & $\mathbf{0}$ \\
\hline valuation & 1529 & 2.70 & & 4.10 & . & 4.77 & $\mathbf{F}$ & 5.04 & $\mathbf{M}$ & 1 \\
\hline valvotomy & 1903 & 3.10 & $\mathbf{M}$ & 1.70 & . & 3.26 & $\mathbf{M}$ & 1.53 & & 0 \\
\hline variation & 1386 & 3.07 & $\mathbf{M}$ & 4.54 & $\mathbf{F}$ & 4.70 & . & 6.84 & & 1 \\
\hline vealy & 1769 & 2.57 & . & 2.67 & . & 3.07 & . & 1.93 & & 0 \\
\hline vending & 1666 & 3.63 & & 5.43 & & 4.04 & . & 6.57 & & 1 \\
\hline ventriloquous & 1713 & 3.70 & $\mathbf{F}$ & 4.60 & $\mathbf{M}$ & 4.17 & & 4.20 & $\mathbf{F}$ & 0 \\
\hline verifiability & 1881 & 2.33 & . & 3.04 & . & 4.90 & . & 4.93 & & 0 \\
\hline vermicule & 1713 & 3.30 & & 1.67 & . & 3.07 & . & 2.37 & $\mathbf{M}$ & 0 \\
\hline vesalian & 1870 & 4.60 & $\mathbf{M}$ & 1.34 & . & 3.10 & . & 1.50 & $\mathbf{M}$ & 0 \\
\hline vicaress & 1613 & 3.37 & $\mathbf{M}$ & 2.23 & . & 3.13 & & 2.60 & $\mathbf{F}$ & 0 \\
\hline vigesimal & 1656 & 2.73 & . & 1.53 & . & 3.43 & $\mathbf{M}$ & 1.53 & & $\mathbf{0}$ \\
\hline vinosity & 1624 & 2.70 & . & 1.67 & . & 3.60 & . & 1.90 & & 0 \\
\hline vintage & 1450 & 3.50 & & 5.07 & . & 5.47 & . & 6.57 & . & 1 \\
\hline virtually & 1430 & 2.23 & $\mathbf{M}$ & 2.83 & & 4.64 & & 6.84 & . & 1 \\
\hline visualize & 1817 & 3.77 & & 5.00 & $\mathbf{F}$ & 5.70 & $\mathbf{F}$ & 6.84 & & 1 \\
\hline vogue & 1571 & 2.70 & & 5.10 & $\mathbf{F}$ & 4.60 & F & 6.67 & & 1 \\
\hline volumeter & 1829 & 5.13 & $\mathbf{M}$ & 2.44 & . & 4.30 & . & 3.47 & $\mathbf{M}$ & 0 \\
\hline vorlaufer & 1961 & 3.70 & & 1.30 & • & 3.33 & . & 1.44 & . & 0 \\
\hline vowing & 1300 & 3.00 & $\mathbf{M}$ & 4.77 & . & 4.67 & . & 5.97 & . & 1 \\
\hline wage & 1183 & 4.80 & $\mathbf{M}$ & 5.57 & & 5.44 & . & 7.00 & & 1 \\
\hline wake & 825 & 4.57 & . & 5.97 & F & 4.77 & . & 6.97 & . & 1 \\
\hline walling & 1000 & 3.00 & & 4.33 & $F$ & 3.34 & . & 3.60 & . & 0 \\
\hline
\end{tabular}


APPENDIX (Continued)

\begin{tabular}{|c|c|c|c|c|c|c|c|c|c|c|}
\hline WORD & DOE & CONC & & IMAG & & GOOD & & FAM & & FREQ \\
\hline walnut & 1050 & 6.70 & & 6.70 & & 5.10 & & 6.97 & & 1 \\
\hline wand & 1200 & 5.97 & & 5.80 & $\mathrm{~F}$ & 4.73 & & 6.50 & $\mathbf{M}$ & 1 \\
\hline wantage & 1609 & 2.77 & $\mathrm{~F}$ & 2.26 & . & 3.50 & $\mathbf{M}$ & 2.54 & $\mathbf{M}$ & 0 \\
\hline ware & 725 & 3.97 & $\mathbf{M}$ & 4.27 & . & 4.13 & 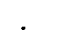 & 5.37 & & 1 \\
\hline warrant & 1225 & 5.07 & $\mathbf{M}$ & 5.47 & & 3.30 & . & 6.90 & & 1 \\
\hline wastable & 1436 & 2.90 & & 4.17 & . & 2.97 & . & 4.93 & $\mathbf{M}$ & 0 \\
\hline water & 897 & 6.73 & 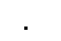 & 6.84 & & 6.20 & . & 7.00 & . & 1 \\
\hline water-soaken & 1783 & 5.27 & & 6.07 & & 2.94 & . & 5.27 & . & 0 \\
\hline waterloo & 1816 & 6.13 & $\mathbf{M}$ & 6.03 & $\mathbf{F}$ & 4.47 & . & 7.00 & . & 1 \\
\hline way & 725 & 2.83 & & 4.00 & . & 4.47 & . & 7.00 & . & 1 \\
\hline wealth & 1250 & 4.17 & $\mathbf{M}$ & 6.17 & & 5.70 & . & 6.87 & . & 1 \\
\hline web & 725 & 6.43 & M & 6.60 & & 3.50 & . & 6.83 & & 1 \\
\hline weftage & 1615 & 3.30 & & 1.33 & $\mathbf{M}$ & 3.14 & . & 1.50 & & 0 \\
\hline well & 825 & 5.53 & $\mathbf{M}$ & 6.20 & $\mathrm{~F}$ & 5.57 & $\mathbf{F}$ & 6.97 & & 1 \\
\hline well-endowed & 1690 & 3.87 & & 5.87 & & 5.23 & . & 6.04 & $\mathbf{M}$ & 0 \\
\hline well-trained & 1611 & 3.15 & $\mathbf{M}$ & 5.50 & $\mathbf{F}$ & 5.77 & . & 6.50 & & 1 \\
\hline westphalian & 1604 & 4.37 & . & 1.60 & $\mathbf{M}$ & 3.60 & . & 2.53 & $\mathbf{M}$ & 0 \\
\hline wheat & 825 & 6.80 & . & 6.67 & $\mathbf{F}$ & 5.07 & . & 7.00 & & 1 \\
\hline whet & 897 & 3.27 & & 2.76 & $\mathbf{M}$ & 3.43 & . & 2.90 & $\mathbf{M}$ & 1 \\
\hline whipcord & 1318 & 5.67 & $\mathbf{M}$ & 5.27 & & 2.70 & & 4.10 & $\mathbf{M}$ & 0 \\
\hline whist & 1382 & 2.93 & $\mathbf{M}$ & 3.77 & $\mathbf{M}$ & 3.80 & $\mathbf{F}$ & 2.90 & . & 1 \\
\hline whitebeam & 1705 & 4.73 & . & 4.43 & & 4.70 & & 3.10 & $\mathrm{~F}$ & 0 \\
\hline whop & 1400 & 3.90 & . & 4.26 & $\mathrm{~F}$ & 3.40 & & 4.70 & & 1 \\
\hline wifish & 1535 & 2.97 & & 2.30 & & 2.97 & & 2.13 & $\mathbf{M}$ & 0 \\
\hline wiggler & 1859 & 4.87 & $\mathbf{M}$ & 5.37 & & 4.34 & $\mathbf{F}$ & 5.57 & . & 0 \\
\hline willer & 1395 & 3.30 & & 2.30 & $\mathrm{~F}$ & 3.87 & . & 3.20 & . & 0 \\
\hline wind-gauge & 1652 & 5.87 & $\mathbf{M}$ & 5.23 & . & 4.50 & . & 4.70 & . & $\mathbf{0}$ \\
\hline winter & 835 & 5.70 & . & 6.50 & & 5.10 & & 7.00 & & 1 \\
\hline wireline & 1858 & 5.43 & & 5.03 & $\mathbf{M}$ & 4.14 & $\mathbf{M}$ & 4.40 & $\mathbf{F}$ & 0 \\
\hline wit & 888 & 3.37 & $\mathbf{M}$ & 4.43 & $\mathbf{M}$ & 5.84 & . & 6.67 & . & 1 \\
\hline within & 1000 & 2.80 & $\mathbf{M}$ & 4.70 & . & 4.40 & . & 6.87 & . & 1 \\
\hline wolf & 725 & 6.73 & . & 6.90 & . & 3.67 & . & 7.00 & . & 1 \\
\hline wood & 725 & 6.33 & & 6.73 & & 5.33 & . & 7.00 & . & 1 \\
\hline work & 725 & 5.10 & . & 6.50 & $\mathrm{~F}$ & 4.77 & $\mathbf{M}$ & 7.00 & . & 1 \\
\hline workfare & 1968 & 3.40 & & 3.40 & . & 4.13 & . & 4.43 & . & 0 \\
\hline worm & 825 & 6.67 & & 6.84 & & 3.23 & . & 6.94 & . & 1 \\
\hline worth & 695 & 2.60 & $\mathbf{M}$ & 4.40 & $\mathrm{~F}$ & 5.40 & . & 6.77 & . & 1 \\
\hline wreak & 725 & 3.73 & M & 4.73 & & 2.30 & $\mathbf{F}$ & 6.14 & . & 1 \\
\hline wring & 888 & 3.83 & $\mathbf{M}$ & 5.20 & $\mathrm{~F}$ & 3.27 & . & 5.20 & & 1 \\
\hline wrongfulness & 1425 & 2.60 & . & 3.90 & $F$ & 2.47 & & 6.07 & & 0 \\
\hline x-radiation & 1896 & 4.70 & . & 4.64 & $\mathbf{F}$ & 2.97 & $\mathbf{M}$ & 5.17 & & 0 \\
\hline yager & 1804 & 3.43 & . & 1.97 & $\mathbf{M}$ & 3.57 & $\mathbf{M}$ & 1.57 & & 0 \\
\hline yegg & 1903 & 3.30 & . & 1.47 & $\mathbf{M}$ & 3.10 & . & 1.50 & & 0 \\
\hline yield & 601 & 3.53 & . & 5.53 & $\mathrm{~F}$ & 4.07 & & 6.93 & . & 1 \\
\hline yolky & 1000 & 3.27 & $\mathbf{M}$ & 4.47 & $\mathrm{~F}$ & 3.57 & F & 3.73 & & 0 \\
\hline your & 825 & 2.33 & . & 3.80 & & 5.10 & $\mathbf{F}$ & 6.87 & & 1 \\
\hline zionism & 1896 & 3.37 & 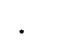 & 3.00 & $\mathbf{M}$ & 3.20 & $\mathbf{M}$ & 3.43 & $\mathbf{M}$ & 1 \\
\hline zizel & 1785 & 3.47 & $\mathrm{~F}$ & 1.83 & $\mathbf{M}$ & 3.73 & & 1.37 & & 0 \\
\hline
\end{tabular}

(Manuscript received August 6, 1988;

revision accepted for publication December 6, 1988.) 